\title{
HARRIS-VIEHMANN CONJECTURE FOR HODGE-NEWTON REDUCIBLE RAPOPORT-ZINK SPACES
}

\author{
SERIN HONG
}

\begin{abstract}
Rapoport-Zink spaces, or more generally local Shimura varieties, are expected to provide geometric realization of the local Langlands correspondence via their $l$-adic cohomology. Along this line is a conjecture by Harris and Viehmann, which roughly says that when the underlying local Shimura datum is not basic, the $l$-adic cohomology of the local Shimura variety is parabolically induced.

We verify this conjecture for Rapoport-Zink spaces which are Hodge type and Hodge-Newton reducible. The main strategy is to embed such a Rapoport-Zink space into an appropriate space of EL type, for which the conjecture is already known to hold by the work of Mantovan.
\end{abstract}

\section{Contents}

1. Introduction

2. Notations and preliminaries

3. Rapoport-Zink spaces of Hodge type

4. Hodge-Newton reducibility and Harris-Viehmann conjecture 12 References

\section{INTRODUCTION}

In [RZ96], Rapoport and Zink constructed formal moduli spaces of $p$-divisible groups which give rise to local analogues of PEL type Shimura varieties. These spaces, which are now called Rapoport-Zink spaces, have played a crucial role in the study of the local Langlands correspondence. Perhaps the most striking example is Harris and Taylor's proof in [HT01] of the local Langlands conjecture for $\mathrm{GL}_{n}$. A key point of their proof is that one can realize the local Langlands correspondence in the l-adic cohomology of Lubin-Tate spaces, which are Rapoport-Zink spaces that parametrize $p$-divisible groups of dimension 1.

The theory of Rapoport-Zink spaces suggests that it should be possible to realize many cases of the local Langlands correspondence via the $l$-adic cohomology of some local analogues of Shimura varieties. Motivated by this, Rapoport and Viehmann in RV14 formulated the idea that there should exist a general theory of local analogues of Shimura varieties, which they called local Shimura varieties. They described a conjectural form of this theory which starts with a group theoretic datum called a local Shimura datum and associates to this datum a tower of analytic spaces which enjoys 
analogous properties to the properties of Shimura varieties. Shortly after this conjectural formulation, Scholze in his Berkeley lectures Sch14 gave a construction of local Shimura varieties in some perfectoid category which he called the category of diamonds. For local Shimura data that arise from Shimura varieties of Hodge type, W. Kim in Kim13 constructed Hodge type Rapoport-Zink spaces which may serve as integral models of the corresponding local Shimura varieties.

There are two key cohomology conjectures, namely the Kottwitz conjecture and the Harris-Viehmann conjecture, which predicts how the $l$-adic cohomology of local Shimura varieties should realize the local Langlands correspondence. The Kottwitz conjecture, originally formulated by Kottwitz and introduced by Rapoport in [Rap94, concerns realization of supercuspidal representations when the underlying local Shimura datum is basic. The Harris-Viehmann conjecture, originally formulated by Harris in Har00] and later modified by Viehmann, gives an inductive formula for the cohomology when the underlying local Shimura datum is not basic.

The primary purpose of this paper is to prove the Harris-Viehmann conjecture for certain local Shimura data that arise from Shimura varieties of Hodge type. We will work in the setting of Rapoport-Zink spaces, as our proof will use previously known results for Rapoport-Zink spaces of PEL type. However, our argument should work as well in the setting of local Shimura varieties constructed by Scholze.

Let us now introduce the notations and terminologies necessary for a precise statement of our result. We fix a prime $p>2$, and set up some standard notations as follows: we write $\overline{\mathbb{F}}_{p}$ and $\overline{\mathbb{Q}}_{p}$ respectively for a fixed algebraic closure of $\mathbb{F}_{p}$ and $\mathbb{Q}_{p} ; \mathbb{Q}_{p}^{\text {un }}$ for the maximal unramified extension of $\mathbb{Q}_{p} ; \mathbb{C}_{p}$ and $\breve{\mathbb{Q}}_{p}$ respectively for the $p$-adic completion of $\overline{\mathbb{Q}}_{p}$ and $\mathbb{Q}_{p}^{\text {un }}$; and $\breve{\mathbb{Z}}_{p}$ for the ring of integers of $\breve{\mathbb{Q}}_{p}$. We also fix an unramified local Shimura datum of Hodge type, which is a tuple $(G,[b],\{\mu\})$ consisting of a connected reductive group $G$ over $\mathbb{Z}_{p}$, a $\sigma$-conjugacy class $[b]$ of elements in $G\left(\check{\mathbb{Q}}_{p}\right)$, and a $G\left(\breve{\mathbb{Z}}_{p}\right)$ conjugacy class $\{\mu\}$ of cocharacters of $G$ satisfying certain axioms (see 3.1.1 for details). Let $E$ denote the field of definition of $\{\mu\}$, which is an unramified finite extension of $\mathbb{Q}_{p}$. With a suitable choice of $b \in[b]$, the datum $(G,[b],\{\mu\})$ gives rise to a $p$-divisible group $X$ over $\overline{\mathbb{F}}_{p}$ with some additional structures induced by the group $G$. Let $J_{b}$ be an algebraic group over $\mathbb{Q}_{p}$ with functor of points

$$
J_{b}(R)=\left\{g \in G\left(R \otimes_{\mathbb{Q}_{p}} \breve{\mathbb{Q}}_{p}\right): g b \sigma(g)^{-1}=b\right\}
$$

for any $\mathbb{Q}_{p}$-algebra $R$.

To the pair $(G, b)$, we associate a Rapoport-Zink space of Hodge type $\mathrm{RZ}_{G, b}$ following W. Kim's construction in [Kim13]. The space $\mathrm{RZ}_{G, b}$ is a formal scheme over Spf $\left(\breve{\mathbb{Z}}_{p}\right)$ whose isomorphism class depends only on the datum $(G,[b],\{\mu\})$. It also has a rigid analytic generic fiber $\mathrm{RZ}_{G, b}^{\text {rig }}$ which is equipped with a tower of étale covers $\mathrm{RZ} Z_{G, b}^{\infty}:=$ $\left\{\mathrm{RZ}_{G, b}^{K_{p}}\right\}$ where $K_{p}$ runs over open compact subgroups of $G\left(\mathbb{Z}_{p}\right)$. The l-adic cohomology groups

$$
H^{i}\left(\mathrm{RZ}_{G, b}^{K_{p}}\right):=H_{c}^{i}\left(\mathrm{RZ}_{G, b}^{K_{p}} \otimes_{\breve{\mathbb{Q}}_{p}} \mathbb{C}_{p}, \mathbb{Q}_{l}\left(\operatorname{dim} \mathrm{RZ}_{G, b}^{K_{p}}\right)\right) \quad \text { for } i>0
$$

fit into a tower $\left\{H^{i}\left(\mathrm{RZ}_{G, b}^{K_{p}}\right)\right\}$ with a natural action of $G\left(\mathbb{Q}_{p}\right) \times W_{E} \times J_{b}\left(\mathbb{Q}_{p}\right)$ where $W_{E}$ is the Weil group of $E$. For an $l$-adic admissible representation $\rho$ of $J_{b}\left(\mathbb{Q}_{p}\right)$, we define 
a virtual representation of $G\left(\mathbb{Q}_{p}\right) \times W_{E}$

$$
H^{\bullet}\left(\mathrm{RZ}_{G, b}^{\infty}\right)_{\rho}:=\sum_{i, j \geq 0}(-1)^{i+j} \underset{K_{p}}{\lim _{\longrightarrow}} \operatorname{Ext}_{J_{b}\left(\mathbb{Q}_{p}\right)}^{j}\left(H^{i}\left(\mathrm{RZ}_{G, b}^{K_{p}}\right), \rho\right) .
$$

We prove the Harris-Viehmann conjecture under the assumption that the datum $(G,[b],\{\mu\})$ is Hodge-Newton reducible. Roughly speaking, this means that the datum $(G,[b],\{\mu\})$ naturally reduces to a local Shimura datum for some Levi subgroup $L$ of $G$. More precisely, there exists a choice of $b \in[b] \cap L\left(\breve{\mathbb{Q}}_{p}\right)$ and $\mu \in\{\mu\}$ which factors through $L$ such that the tuple $(L,[b],\{\mu\})$ is an unramified local Shimura datum of Hodge type (see 4.1 .4 for details).

Now we can state our main result as follows:

Theorem. Assume that the unramified local Shimura datum of Hodge type $(G,[b],\{\mu\})$ is Hodge-Newton reducible with respect to a parabolic subgroup $P$ of $G$ with Levi factor L. Choose $b \in[b] \cap L\left(\breve{\mathbb{Q}}_{p}\right)$ which gives rise to a $p$-divisible group over $\overline{\mathbb{F}}_{p}$ with additional structures induced by $L$. For any admissible $\overline{\mathbb{Q}}_{l}$-representation $\rho$ of $J\left(\mathbb{Q}_{p}\right)$, we have the following equality of virtual representations of $G\left(\mathbb{Q}_{p}\right) \times W_{E}$ :

$$
H^{\bullet}\left(R Z_{G, b}^{\infty}\right)_{\rho}=\operatorname{Ind} d_{P\left(\mathbb{Q}_{p}\right)}^{G\left(\mathbb{Q}_{p}\right)} H^{\bullet}\left(R Z_{L, b}^{\infty}\right)_{\rho} .
$$

In particular, the virtual representation $H^{\bullet}\left(R Z_{G, b}^{\infty}\right)_{\rho}$ contains no supercuspidal representations of $G\left(\mathbb{Q}_{p}\right)$.

Let us record some previously known results on the Harris-Viehmann conjecture. The earliest result of this form is Boyer's work in Boy99 for Drinfeld's modular varieties. For Rapoport-Zink spaces of PEL type, Mantovan in Man08 and Shen in Sh13] verified the conjecture assuming Hodge-Newton reducibility. For local Shimura varieties constructed by Scholze, Hansen in [Han16] proves the conjecture for $G=\mathrm{GL}_{n}$ also under the Hodge-Newton reducibility assumption.

We now briefly sketch our proof of the theorem. Our overall strategy is to prove that the rigid analytic generic fiber of the space $\mathrm{RZ}_{G, b}$ is "parabolically induced" from the rigid analytic generic fiber of $\mathrm{RZ}_{L, b}$. More precisely, we will construct an analogue of Rapoport-Zink space $\mathrm{RZ}_{P, b}$ associated to the parabolic subgroup $P$ and prove the following lemma:

Lemma. The rigid analytic generic fibers of $R Z_{G, b}, R Z_{P, b}$ and $R Z_{L, b}$ fit into a diagram

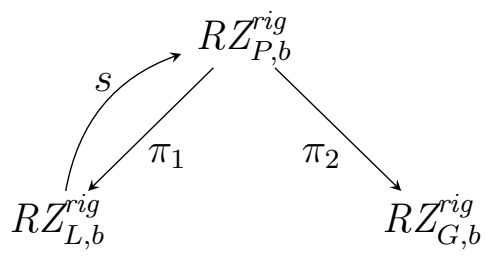

such that

(i) $s$ is a closed immersion,

(ii) $\pi_{1}$ is a fibration in balls,

(iii) $\pi_{2}$ is an isomorphism. 
After establishing this lemma, we deduce the theorem by comparing the cohomology of the spaces $\mathrm{RZ}_{L, b}$ and $\mathrm{RZ}_{G, b}$ with the cohomology of $\mathrm{RZ}_{P, b}$.

This strategy originated in Mantovan's proof in [Man08, and also appeared in the work of Shen in [Sh13] and Hansen in [Han16]. However, details of our argument will be different from those in the aforementioned works.

The main ingredient of our argument is the notion of EL realization developed by the author in [Hong16]. An EL realization of the datum $(G,[b],\{\mu\})$ is an embedding of $(G,[b],\{\mu\})$ into a local Shimura datum of EL type $(\widetilde{G},[b],\{\mu\})$ which is Hodge-Newton reducible with respect to a parabolic subgroup $\widetilde{P}$ of $\widetilde{G}$ with Levi factor $\widetilde{L}$ such that $P=\widetilde{P} \cap G$ and $L=\widetilde{L} \cap G$. By functoriality of Hodge type Rapoport-Zink spaces, an EL realization of $(G,[b],\{\mu\})$ induces a closed embedding

$$
\mathrm{RZ}_{G, b} \hookrightarrow \mathrm{RZ}_{\widetilde{G}, b} .
$$

Over the EL type Rapoport-Zink space $\mathrm{RZ}_{\widetilde{G}, b}$, Mantovan constructed an analogue of Rapoport-Zink space $\mathrm{RZ}_{\widetilde{P}, b}$ associated to $\widetilde{P}$. We will use the results from [Hong16] to prove that the pull back of $\mathrm{RZ}_{\widetilde{P}, b}$ over $\mathrm{RZ}_{G, b}$ is the desired space $\mathrm{RZ} Z_{P, b}$ that yields the diagram in the lemma.

We now give an overview of the structure of this paper. In section 2, we introduce general notations and recall some group theoretic preliminaries. In section 3, we review W. Kim's construction of Rapoport-Zink spaces of Hodge type. In section 4, we state and prove our main theorem.

Acknowledgments. I would like to express my deepest gratitude to Elena Mantovan. This study would have never been possible without her previous work for EL/PEL cases and her numerous helpful suggestions.

\section{Notations AND PRELIMINARIES}

\subsection{General notations.}

Throughout this paper, we use the following standard notations:

- $\overline{\mathbb{F}}_{p}$ is a fixed algebraic closure of $\mathbb{F}_{p}$;

- $\overline{\mathbb{Q}}_{p}$ is a fixed algebraic closure of $\mathbb{Q}_{p}$;

- $\mathbb{Q}_{p}^{\text {un }}$ is the maximal unramified extension of $\mathbb{Q}_{p}$ in $\overline{\mathbb{Q}}_{p}$;

- $\mathbb{C}_{p}$ is the $p$-adic completion of $\overline{\mathbb{Q}}_{p}$;

- $\breve{\mathbb{Q}}_{p}$ is the $p$-adic completion of $\mathbb{Q}_{p}^{\text {un }}$;

- $\breve{\mathbb{Z}}_{p}$ is the ring of integers of $\breve{\mathbb{Q}}_{p}$.

In addition, we denote by $\sigma$ the Frobenius automorphism of $\overline{\mathbb{F}}_{p}$ and also its lift to $\breve{\mathbb{Z}}_{p}$ and $\breve{\mathbb{Q}}_{p}$.

Given a Noetherian ring $R$ and a free $R$-module $\Lambda$, we denote by $\Lambda^{\otimes}$ the direct sum of all the $R$-modules which can be formed from $\Lambda$ using the operations of taking duals, tensor products, symmetric powers and exterior powers. An element of $\Lambda^{\otimes}$ is called a tensor over $\Lambda$. Note that there is a natural identification $\Lambda^{\otimes} \simeq\left(\Lambda^{*}\right)^{\otimes}$ where $\Lambda^{*}$ is 
the dual $R$-module of $\Lambda$. Any isomorphism $\Lambda \stackrel{\sim}{\rightarrow} \Lambda^{\prime}$ of free $R$-modules of finite rank naturally induces an isomorphism $\Lambda^{\otimes} \stackrel{\sim}{\rightarrow}\left(\Lambda^{\prime}\right)^{\otimes}$.

For a $p$-divisible group $X$ over a $\mathbb{Z}_{p}$-scheme $S$, we write $\mathbb{D}(X)$ for its (contravariant) Dieudonné module and $\mathrm{Fil}^{1}(\mathbb{D}(X)) \subset \mathbb{D}(X)_{S}$ for its Hodge filtration. We generally denote by $F$ the Frobenius map on $\mathbb{D}(X)$.

\subsection{Group theoretic preliminaries.}

2.2.1. Let $G$ be a connected reductive group over $\mathbb{Z}_{p}$. We write $\operatorname{Rep}_{\mathbb{Z}_{p}}(G)$ for the category of finite rank $G$-representations of over $\mathbb{Z}_{p}$, and $\operatorname{Rep}_{\mathbb{Q}_{p}}(G)$ for the category of finite dimensional $G_{\mathbb{Q}_{p}}$-representations over $\mathbb{Q}_{p}$.

Let $R$ be a $\mathbb{Z}_{p}$-algebra, and let $\lambda: \mathbb{G}_{m} \rightarrow G_{R}$ be a cocharacter. We denote by $\{\lambda\}_{G}$, or usually by $\{\lambda\}$ if there is no risk of confusion, the $G(R)$-conjugacy class of $\lambda$. When $R=\breve{\mathbb{Z}}_{p}$, we have a bijection

$$
\operatorname{Hom}_{\breve{\mathbb{Z}}_{p}}\left(\mathbb{G}_{m}, G_{\breve{\mathbb{Z}}_{p}}\right) / G\left(\breve{\mathbb{Z}}_{p}\right) \cong \operatorname{Hom}_{\breve{\mathbb{Q}}_{p}}\left(\mathbb{G}_{m}, G_{\breve{\mathbb{Q}}_{p}}\right) / G\left(\breve{\mathbb{Q}}_{p}\right) \stackrel{\sim}{\longrightarrow} G\left(\breve{\mathbb{Z}}_{p}\right) \backslash G\left(\breve{\mathbb{Q}}_{p}\right) / G\left(\breve{\mathbb{Z}}_{p}\right)
$$

induced by $\{\lambda\} \mapsto G\left(\breve{\mathbb{Z}}_{p}\right) \lambda(p) G\left(\breve{\mathbb{Z}}_{p}\right)$; in fact, the first bijection comes from the fact that $G$ is split over $\breve{\mathbb{Z}}_{p}$, whereas the second bijection is the Cartan decomposition.

Let $\Lambda \in \operatorname{Rep}_{\mathbb{Z}_{p}}(G)$ be a faithful $G$-representation over $\mathbb{Z}_{p}$. By [Ki10], Proposition 1.3.2, we can choose a finite family of tensors $\left(s_{i}\right)_{i \in I}$ on $\Lambda$ such that $G$ is the pointwise stabilizer of the $s_{i}$; i.e., for any $\mathbb{Z}_{p}$-algebra $R$ we have

$$
G(R)=\left\{g \in \mathrm{GL}\left(\Lambda \otimes_{\mathbb{Z}_{p}} R\right): g\left(s_{i} \otimes 1\right)=s_{i} \otimes 1 \text { for all } i \in I\right\} .
$$

We say that a grading $\operatorname{gr} \bullet\left(\Lambda_{R}\right)$ is induced by $\lambda$ if the following conditions are satisfied:

(i) the $\mathbb{G}_{m}$-action on $\Lambda_{R}$ via $\lambda$ leaves each grading stable,

(ii) the resulting $\mathbb{G}_{m}$-action on $\operatorname{gr}^{i}\left(\Lambda_{R}\right)$ is given by

$$
\mathbb{G}_{m} \stackrel{z \mapsto z^{-i}}{\longrightarrow} \mathbb{G}_{m} \stackrel{z \mapsto z \cdot \mathrm{id}}{\longrightarrow} \mathrm{GL}\left(\mathrm{gr}^{i}\left(\Lambda_{R}\right)\right)
$$

Let $S$ be an $R$-scheme, and $\mathscr{E}$ a vector bundle on $S$. For a finite family of global sections $\left(t_{i}\right)$ of $\mathscr{E}^{\otimes}$, we define the following scheme over $S$

$$
\mathcal{P}_{S}:=\operatorname{Isom}_{\mathcal{O}_{S}}\left(\left[\mathscr{E},\left(t_{i}\right)\right],\left[\Lambda \otimes_{R} \mathcal{O}_{S},\left(s_{i} \otimes 1\right)\right]\right)
$$

In other words, $\mathcal{P}_{S}$ classifies isomorphisms of vector bundles $\mathscr{E} \cong \Lambda \otimes_{R} \mathcal{O}_{S}$ which match $\left(t_{i}\right)$ and $\left(s_{i} \otimes 1\right)$.

Let $\mathrm{Fil}^{\bullet}(\mathscr{E})$ be a filtration of $\mathscr{E}$. When $\mathcal{P}_{S}$ is a trivial $G$-torsor, we say that $\mathrm{Fil}^{\bullet}(\mathscr{E})$ is a $\{\lambda\}$-filtration with respect to $\left(t_{i}\right)$ if there exists an isomorphism $\mathscr{E} \cong \Lambda \otimes_{R} \mathcal{O}_{S}$, matching $\left(t_{i}\right)$ and $\left(1 \otimes s_{i}\right)$, which takes Fil ${ }^{\bullet}(\mathscr{E})$ to a filtration of $\Lambda \otimes_{R} \mathcal{O}_{S}$ induced by $g \lambda g^{-1}$ for some $g \in G(R)$. More generally, when $\mathcal{P}_{S}$ a $G$-torsor, we say that $\mathrm{Fil}^{\bullet}(\mathscr{E})$ is a $\{\lambda\}$-filtration with respect to $\left(t_{i}\right)$ if it is étale-locally a $\{\lambda\}$-filtration. 
2.2.2. We say that $b, b^{\prime} \in G\left(\breve{\mathbb{Q}}_{p}\right)$ are $\sigma$-conjugate if $b^{\prime}=g b \sigma(g)^{-1}$ for some $g \in G\left(\breve{\mathbb{Q}}_{p}\right)$. We denote by $B(G)$ the set of all $\sigma$-conjugacy classes in $G\left(\breve{\mathbb{Q}}_{p}\right)$. We write $[b]_{G}$, or simply $[b]$ when there is no risk of confusion, for the $\sigma$-conjugacy class of $b \in G\left(\breve{\mathbb{Q}}_{p}\right)$.

Let us now fix a $\sigma$-conjugacy class $[b]$ in $B(G)$ and choose an element $b \in[b]$. We define a group valued functor $J_{b}$ on the category of $\mathbb{Q}_{p}$-algebras by setting for any $\mathbb{Q}_{p}$-algebra $R$

$$
J_{b}(R):=\left\{g \in G\left(R \otimes_{\mathbb{Q}_{p}} \breve{\mathbb{Q}}_{p}\right): g b \sigma(g)^{-1}=b\right\} .
$$

This functor is represented by an algebraic group over $\mathbb{Q}_{p}$ which is an inner form of some Levi subgroup of $G_{\mathbb{Q}_{p}}$ (see [RZ96], Corollary 1.14.). The isomorphism class of $J_{b}$ does not depend on the choice $b \in[b]$ since any $g \in G\left(\breve{\mathbb{Q}}_{p}\right)$ induces an isomorphism $J_{b} \cong J_{g b \sigma(g)^{-1}}$ via conjugation.

By an $F$-isocrystal over $\overline{\mathbb{F}}_{p}$, we mean a vector space over $\breve{\mathbb{Q}}_{p}$ equipped with a $\sigma$-linear automorphism $F$. Given a $G_{\mathbb{Q}_{p}}$-representation $\rho: G_{\mathbb{Q}_{p}} \longrightarrow \operatorname{GL}(V)$ over $\mathbb{Q}_{p}$, we set $N_{b}(\rho)$ to be the $\breve{\mathbb{Q}}_{p}$-vector space $V \otimes_{\mathbb{Q}_{p}} \breve{\mathbb{Q}}_{p}$ with $F=\rho(b) \circ(1 \otimes \sigma)$. Then $N_{b}$ defines a functor from $\operatorname{Rep}_{\mathbb{Q}_{p}}(G)$ to the category of $F$-isocrystals over $\overline{\mathbb{F}}_{p}$. One easily checks that another choice $b^{\prime} \in[b]$ gives an isomorphic functor $N_{b^{\prime}}$.

\section{RAPOPORT-ZinK SPACES OF Hodge type}

In this section, we discuss the construction and key properties of Rapoport-Zink spaces of Hodge type, following Kim13.

\subsection{Construction.}

3.1.1. An unramified local Shimura datum of Hodge type is a tuple $(G,[b],\{\mu\})$ where

- $G$ is a connected reductive group over $\mathbb{Z}_{p}$;

- $[b]$ is a $\sigma$-conjugacy class of $G\left(\breve{\mathbb{Q}}_{p}\right)$;

- $\{\mu\}$ is a $G\left(\breve{\mathbb{Z}}_{p}\right)$-conjugacy class of cocharacters of $G$,

which satisfy the following conditions:

(i) $\{\mu\}$ is minuscule,

(ii) $[b] \cap G\left(\breve{\mathbb{Z}}_{p}\right) \mu(p) G\left(\breve{\mathbb{Z}}_{p}\right)$ is not empty for some (and hence for all) $\mu \in\{\mu\}$,

(iii) there exists a faithful $G$-representation $\Lambda \in \operatorname{Rep}_{\mathbb{Z}_{p}}(G)$ (with its dual $\Lambda^{*}$ ) such that, for some $b \in[b] \cap G\left(\breve{\mathbb{Z}}_{p}\right) \mu(p) G\left(\breve{\mathbb{Z}}_{p}\right)$, we have a $\breve{\mathbb{Z}}_{p}$-lattice

$$
M \simeq \Lambda^{*} \otimes_{\mathbb{Z}_{p}} \breve{\mathbb{Z}}_{p} \subset N_{b}\left(\Lambda^{*} \otimes_{\mathbb{Z}_{p}} \mathbb{Q}_{p}\right)
$$

with the property $p M \subset F M \subset M$.

Note that the set $G\left(\breve{\mathbb{Z}}_{p}\right) \mu(p) G\left(\breve{\mathbb{Z}}_{p}\right)$ is independent of the choice $\mu \in\{\mu\}$ as explained in 2.2 .1

Condition (iii) implies that for all $b \in[b] \cap G\left(\breve{\mathbb{Z}}_{p}\right) \mu(p) G\left(\breve{\mathbb{Z}}_{p}\right)$, we can find a $\breve{\mathbb{Z}}_{p}$-lattice

$$
M \simeq \Lambda^{*} \otimes_{\mathbb{Z}_{p}} \breve{\mathbb{Z}}_{p} \subset N_{b}\left(\Lambda^{*} \otimes_{\mathbb{Z}_{p}} \mathbb{Q}_{p}\right)
$$


with the property $p M \subset F M \subset M$. In fact, existence of $M$ is equivalent to the condition that the linearization of $F$ has an integer matrix representation with respect to some basis, which depends only on $[b]$.

Let us explain how the above definition is related to the definition of local Shimura data given by Rapoport and Viehmann in [RV14, Definition 5.1. Since $G$ is split over $\breve{\mathbb{Z}}_{p}$, we may regard $\{\mu\}$ as a geometric conjugacy class of cocharacters of $G$. Then by the work of Kottwitz-Rapoport [KR03, Lucarelli [Lu04] and Gashi Ga10, we can state the condition (ii) as $[b] \in B\left(G_{\mathbb{Q}_{p}},\{\mu\}\right)$ where $B\left(G_{\mathbb{Q}_{p}},\{\mu\}\right)$ is the Kottwitz set defined in [?]. Hence the tuple $\left(G_{\mathbb{Q}_{p}},[b],\{\mu\}\right)$ is a local Shimura datum as defined in [RV14], Definition 5.1.

Lemma 3.1.2. Let $(G,[b],\{\mu\})$ be an unramified local Shimura datum of Hodge type.

(1) For any unramified local Shimura datum of Hodge type $\left(G^{\prime},\left[b^{\prime}\right],\left\{\mu^{\prime}\right\}\right)$, the tuple $\left(G \times G^{\prime},\left[b, b^{\prime}\right],\left\{\mu, \mu^{\prime}\right\}\right)$ is also an unramified local Shimura datum of Hodge type.

(2) For any homomorphism $f: G \longrightarrow G^{\prime}$ of connected reductive group over $\mathbb{Z}_{p}$, the tuple $\left(G^{\prime},[f(b)],\{f \circ \mu\}\right)$ is an unramified local Shimura datum of Hodge type.

Proof. This is an easy consequence of definition.

3.1.3. For the rest of this section, we fix our unramified local Shimura datum of Hodge type $(G,[b],\{\mu\})$ and also a faithful $G$-representation $\Lambda \in \operatorname{Rep}_{\mathbb{Z}_{p}}(G)$ in the condition (iii) of 3.1.1. By Lemma 3.1.2, we obtain a morphism of unramified local Shimura data of Hodge type

$$
(G,[b],\{\mu\}) \longrightarrow\left(\mathrm{GL}(\Lambda),[b]_{\mathrm{GL}(\Lambda)},\{\mu\}_{\mathrm{GL}(\Lambda)}\right) .
$$

Let us now choose an element $b \in[b] \cap G\left(\breve{\mathbb{Z}}_{p}\right) \mu(p) G\left(\breve{\mathbb{Z}}_{p}\right)$ and take $M \simeq \Lambda^{*} \otimes_{\mathbb{Z}_{p}} \breve{\mathbb{Z}}_{p}$ as in the condition (iii) of 3.1.1. We also choose a finite family of tensors $\left(s_{i}\right)_{i \in I}$ on $\Lambda$ as in 2.2.1. Then $M \simeq \Lambda^{*} \otimes_{\mathbb{Z}_{p}} \breve{\mathbb{Z}}_{p}$ is equipped with tensors $\left(t_{i}\right):=\left(s_{i} \otimes 1\right)$, which are $F$-invariant since the linearization of $F$ on $M[1 / p]=N_{b}\left(\Lambda^{*} \otimes_{\mathbb{Z}_{p}} \mathbb{Q}_{p}\right)$ is given by $b \in G\left(\breve{\mathbb{Q}}_{p}\right)$.

Take $X$ to be a $p$-divisible group over $\overline{\mathbb{F}}_{p}$ with $\mathbb{D}(X)=M$. Then the Hodge filtration $\mathrm{Fil}^{1}(\mathbb{D}(X)) \subset \mathbb{D}(X)$ is a $\left\{\sigma^{-1}\left(\mu^{-1}\right)\right\}$-filtration with respect to $\left(t_{i}\right)$ (see [Kim13, Lemma 2.5.7 and Remark 2.5.8.). We may regard the tensors $\left(t_{i}\right)$ as additional structures on $X$ induced by the group $G$. We will often refer to these additional structures as $G$ structure on $X$. We will write $\underline{X}:=\left(X,\left(t_{i}\right)\right)$ to indicate the $p$-divisible group $X$ with $G$-structure.

3.1.4. Let Nilp $_{\breve{\mathbb{Z}}_{p}}$ denote the category of $\breve{\mathbb{Z}}_{p}$-algebra where $p$ is nilpotent. For any $R \in \operatorname{Nilp}_{\breve{Z}_{p}}$ we set $\mathrm{RZ}_{b}(R)$ to be the set of isomorphism classes of pairs $(\mathcal{X}, \iota)$ where

- $\mathcal{X}$ is a $p$-divisible group over $R$;

- $\iota: X_{R / p} \longrightarrow \mathcal{X}_{R / p}$ is a quasi-isogeny, i.e., an invertible global section of $\operatorname{Hom}\left(X_{R / p}, \mathcal{X}_{R / p}\right) \otimes_{\mathbb{Z}} \mathbb{Q}$.

Then $\mathrm{RZ}_{b}$ defines a covariant set-valued functor on $\mathrm{Nil}_{\breve{\mathbb{Z}}_{p}}$, which does not depend on the choice of $b \in[b] \cap G\left(\breve{\mathbb{Z}}_{p}\right) \mu(p) G\left(\breve{\mathbb{Z}}_{p}\right)$ up to isomorphism. Rapoport and Zink in [RZ96] proved that the functor $\mathrm{RZ}_{b}$ is represented by a formal scheme which is locally formally 
of finite type and formally smooth over $\breve{\mathbb{Z}}_{p}$. We write $\mathrm{RZ}_{b}$ also for the representing formal scheme, and $\mathcal{X}_{\mathrm{GL}, b}$ for the universal $p$-divisible group over $\mathrm{RZ}_{b}$.

3.1.5. Given a pair $(\mathcal{X}, \iota) \in \mathrm{RZ}_{b}(R)$ with $R \in \mathrm{Nilp}_{\breve{\mathbb{Z}}_{p}}$, we have an isomorphism

$$
\mathbb{D}(\iota): \mathbb{D}\left(\mathcal{X}_{R / p}\right)[1 / p] \stackrel{\sim}{\longrightarrow} \mathbb{D}\left(X_{R / p}\right)[1 / p]
$$

induced by $\iota$. We write $\left(t_{\mathcal{X}, i}\right)$ for the inverse image of the tensors $\left(t_{i}\right)_{R}$ under this isomorphism.

Let Nilp $\breve{\mathbb{Z}}_{p}^{\text {sm }}$ denote the full subcategory of Nilp $\breve{\mathbb{Z}}_{p}$ consisting of formally smooth and formally finitely generated algebra over $\breve{\mathbb{Z}}_{p} / p^{m}$ for some positive integer $m$. For any $R \in \operatorname{Nilp}_{\widetilde{\mathbb{Z}}_{p}}^{\text {sm }}$, we define the set $\mathrm{RZ}_{G, b}^{\left(s_{i}\right)}(R) \subset \operatorname{Hom}_{\breve{\mathbb{Z}}_{p}}\left(\operatorname{Spf}(R), \mathrm{RZ}_{b}\right)$ as follows: for a morphism $f: \operatorname{Spf}(R) \rightarrow \mathrm{RZ}_{b}$ and a $p$-divisible group $\mathcal{X}$ over $\operatorname{Spec}(R)$ which pulls back to $f^{*} \mathcal{X}_{\mathrm{GL}, b}$ over $\operatorname{Spf}(R)$, we have $f \in \mathrm{RZ}_{G, b}^{\left(s_{i}\right)}(R)$ if and only if there exists a (unique) family of tensors $\left(\mathbf{t}_{i}\right)$ on $\mathbb{D}(\mathcal{X})$ with the following properties:

(i) for some ideal of definition $J$ of $R$ containing $p$, the pull-back of $\left(\mathbf{t}_{i}\right)$ over $R / J$ agrees with the pull-back of $\left(t_{\mathcal{X}, i}\right)$ over $R / J$,

(ii) for a $p$-adic lift $\mathcal{R}$ of $R$ which is formally smooth over $\breve{\mathbb{Z}}_{p}$, the $\mathcal{R}$-scheme

$$
\mathcal{P}_{\mathcal{R}}:=\operatorname{Isom}_{\mathcal{R}}\left(\left[\mathbb{D}(\mathcal{X})_{\mathcal{R}},\left(\mathbf{t}_{i}\right)_{\mathcal{R}}\right],\left[\Lambda^{*} \otimes_{\mathbb{Z}_{p}} \mathcal{R},\left(s_{i} \otimes 1\right)\right]\right)
$$

defined as in 2.2 .1 is a $G$-torsor,

(iii) the Hodge filtration of $\mathcal{X}$ is a $\left\{\sigma^{-1}\left(\mu^{-1}\right)\right\}$-filtration with respect to $\left(\mathbf{t}_{i}\right)$.

Then $\mathrm{RZ}_{G, b}^{\left(s_{i}\right)}$ defines a set-valued functor on $\mathrm{Nilp}_{\widetilde{\mathbb{Z}}_{p}}^{\mathrm{sm}}$.

3.1.6. Let us give a concrete description of the set $\mathrm{RZ}_{G, b}^{\left(s_{i}\right)}\left(\overline{\mathbb{F}}_{p}\right)$. Consider a pair $(\mathcal{X}, \iota) \in$ $\mathrm{RZ}_{b}\left(\overline{\mathbb{F}}_{p}\right)$ with a family of tensors $\left(\mathbf{t}_{i}\right)$ on $\mathbb{D}(\mathcal{X})$. Then $\left(\mathbf{t}_{i}\right)$ has the property (i) of 3.1 .5 if and only if it is matched with the family $\left(t_{i}\right)$ under the isomorphism

$$
\mathbb{D}(\iota): \mathbb{D}(\mathcal{X})[1 / p] \stackrel{\sim}{\longrightarrow} \mathbb{D}(X)[1 / p]
$$

induced by $\iota$. In addition, it satisfies the properties (ii) and (iii) of 3.1.5 if and only if $\left(\mathcal{X},\left(\mathbf{t}_{i}\right)\right)$ is a $p$-divisible group with $G$-structure that arises from the datum $(G,[b],\{\mu\})$. Hence the set $\mathrm{RZ}_{G, b}^{\left(s_{i}\right)}\left(\overline{\mathbb{F}}_{p}\right)$ classifies the isomorphism classes of tuples $\left(\mathcal{X},\left(\mathbf{t}_{i}\right), \iota\right)$ where

- $\left(\mathcal{X},\left(\mathbf{t}_{i}\right)\right)$ is a $p$-divisible group over $\overline{\mathbb{F}}_{p}$ with $G$-structure;

- $\iota: X \longrightarrow \mathcal{X}$ is a quasi-isogeny such that the induced isomorphism $\mathbb{D}(\mathcal{X})[1 / p] \stackrel{\sim}{\longrightarrow}$ $\mathbb{D}(X)[1 / p]$ matches $\left(\mathbf{t}_{i}\right)$ with $\left(t_{i}\right)$.

Proposition 3.1.7 ([Kim13], Theorem 4.9.1.). Assume that $p>2$. Then there exists a closed formal subscheme $R Z_{G, b} \subset R Z_{b}$, which is formally smooth over $\breve{\mathbb{Z}}_{p}$ and represents the functor $R Z_{G, b}^{\left(s_{i}\right)}$ for any choice of the tensors $\left(s_{i}\right)$ in 3.1.3. Moreover, the isomorphism class of the formal scheme $R Z_{G, b}$ depends only on the datum $(G,[b],\{\mu\})$.

We let $\mathcal{X}_{G, b}$ denote the "universal $p$-divisible group" over $\mathrm{RZ}_{G, b}$, obtained by taking the pull-back of $\mathcal{X}_{\mathrm{GL}, b}$. Then we obtain a family of "universal tensors" (t $\left.\mathbf{t}_{i}^{\text {univ }}\right)$ on $\mathbb{D}\left(\mathcal{X}_{G, b}\right)$ by applying the universal property to an open affine covering of $\mathrm{RZ}_{G, b}$. 
Example 3.1.8. Consider the case $G=\operatorname{Res}_{\mathscr{O} \mid \mathbb{Z}_{p}} \mathrm{GL}_{n}$ where $\mathscr{O}$ is the ring of integers of some finite unramified extension of $\mathbb{Q}_{p}$. In this case, choosing a family of tensors $\left(s_{i}\right)$ on $\Lambda$ as in 3.1 .3 is equivalent to choosing a $\mathbb{Z}_{p}$-basis of $\mathscr{O}$. Then the family of tensors $\left(t_{i}\right)$ encodes an action of $\mathscr{O}$ on $M$ and thus on $X$. Hence $\underline{X}=\left(X,\left(t_{i}\right)\right)$ can be identified with a $p$-divisible group $X$ with an action of $\mathscr{O}$.

In this setting, the construction of $\mathrm{RZ}_{G, b}$ agrees with the construction of RapoportZink spaces of EL type in [RZ96] (see [Kim13], Proposition 4.7.1.). In other words, for any $R \in \operatorname{Nilp}_{\breve{\mathbb{Z}}_{p}}$ the set $\mathrm{RZ}_{G, b}(R)$ classifies the isomorphism classes of pairs $(\mathcal{X}, \iota)$ where

- $\mathcal{X}$ is a $p$-divisible group over $R$, endowed with an action of $\mathscr{O}$ such that

$$
\operatorname{det}_{R}(a, \operatorname{Lie}(\mathcal{X}))=\operatorname{det}\left(a, \operatorname{Fil}^{0}(\mathbb{D}(X))_{\breve{Q}_{p}}\right) \quad \text { for all } a \in \mathscr{O},
$$

- $\iota: X_{R / p} \rightarrow \mathcal{X}_{R / p}$ is a quasi-isogeny which commutes with the action of $\mathscr{O}$.

\subsection{Functorial properties.}

For the rest of this section, we assume that $p>2$ and take $\mathrm{RZ}_{G, b}$ as in Proposition 3.1.7.

Proposition 3.2.1 ([Kim13], Theorem 4.9.1.). Let $\left(G^{\prime},\left[b^{\prime}\right],\left\{\mu^{\prime}\right\}\right)$ be another unramified local Shimura datum of Hodge type, and choose $b^{\prime} \in\left[b^{\prime}\right] \cap G\left(\breve{\mathbb{Z}}_{p}\right) \mu^{\prime}(p) G\left(\breve{\mathbb{Z}}_{p}\right)$ that gives rise to a p-divisible group over $\overline{\mathbb{F}}_{p}$ with $G^{\prime}$-structure as in 3.1.3.

(1) The natural morphism $R Z_{b} \times_{\text {Spf }\left(\breve{\mathbb{Z}}_{p}\right)} R Z_{b^{\prime}} \longrightarrow R Z_{\left(b, b^{\prime}\right)}$, defined by the product of p-divisible groups with quasi-isogeny, induces an isomorphism

$$
R Z_{G, b} \times_{S p f\left(\breve{\mathbb{Z}}_{p}\right)} R Z_{G^{\prime}, b^{\prime}} \stackrel{\sim}{\longrightarrow} R Z_{G \times G^{\prime},\left(b, b^{\prime}\right)}
$$

(2) For any homomorphism $f: G \longrightarrow G^{\prime}$ with $f(b)=b^{\prime}$, there exists an induced morphism

$$
R Z_{G, b} \longrightarrow R Z_{G^{\prime}, b^{\prime}}
$$

which is a closed embedding if $f$ is a closed embedding.

3.2.2. We want to describe the functorial properties in Proposition 3.2 .1 on the set of $\overline{\mathbb{F}}_{p}$-valued points. For this, we introduce the set

$$
X_{\{\mu\}}^{G}([b]):=\left\{g \in G\left(\breve{\mathbb{Q}}_{p}\right) / G\left(\breve{\mathbb{Z}}_{p}\right) \mid g b \sigma(g)^{-1} \in G\left(\breve{\mathbb{Z}}_{p}\right) \mu(p) G\left(\breve{\mathbb{Z}}_{p}\right)\right\}
$$

which is clearly independent of our choice of $b \in[b]$ up to bijection. The set $X_{\{\mu\}}^{G}([b])$ is called the affine Deligne-Lusztig set associated to the datum $(G,[b],\{\mu\})$. As explained in [Kim13, 4.8, we have a natural bijection

$$
X_{\{\mu\}}^{G}([b]) \stackrel{\sim}{\longrightarrow} \mathrm{RZ}_{G, b}\left(\overline{\mathbb{F}}_{p}\right) .
$$

Let us now consider another unramified local Shimura datum $\left(G^{\prime},\left[b^{\prime}\right],\left\{\mu^{\prime}\right\}\right)$ and choose $b^{\prime} \in\left[b^{\prime}\right] \cap G\left(\breve{\mathbb{Z}}_{p}\right) \mu^{\prime}(p) G\left(\breve{\mathbb{Z}}_{p}\right)$ as in Proposition 3.2.1. Then on the set of $\overline{\mathbb{F}}_{p^{-}}$ valued points, the morphism in (1) of Proposition 3.2.1 gives a map

$$
X_{\{\mu\}}^{G}([b]) \times X_{\left\{\mu^{\prime}\right\}}^{G^{\prime}}\left(\left[b^{\prime}\right]\right) \stackrel{\sim}{\longrightarrow} X_{\left\{\mu, \mu^{\prime}\right\}}^{G \times G^{\prime}}\left(\left[b, b^{\prime}\right]\right)
$$


which maps $\left(g G\left(\breve{\mathbb{Z}}_{p}\right), g^{\prime} G^{\prime}\left(\breve{\mathbb{Z}}_{p}\right)\right)$ to $\left(g, g^{\prime}\right)\left(G \times G^{\prime}\right)\left(\breve{\mathbb{Z}}_{p}\right)$. For any homomorphism $f$ : $G \longrightarrow G^{\prime}$ with $f(b)=b^{\prime}$, the morphism in $(2)$ of Proposition 3.2.1 yields a map

$$
X_{\{\mu\}}^{G}([b]) \longrightarrow X_{\{f \circ \mu\}}^{G^{\prime}}([f(b)])
$$

which maps $g G\left(\breve{\mathbb{Z}}_{p}\right)$ to $f(g) G^{\prime}\left(\breve{\mathbb{Z}}_{p}\right)$.

3.2.3. We now describe the functorial properties in Proposition 3.2.1 on the formal completions at an $\overline{\mathbb{F}}_{p}$-valued point. Let $x$ be a point in $\mathrm{RZ}_{G, b}\left(\overline{\mathbb{F}}_{p}\right)$, and write $\left(X_{x},\left(t_{x, i}\right), \iota_{x}\right)$ for the corresponding tuple under the description of $\mathrm{RZ}_{G, b}\left(\overline{\mathbb{F}}_{p}\right)$ in 3.1 .6 . We denote by $\left(\widehat{\mathrm{RZ}_{G, b}}\right)_{x}$ the formal completion of $\mathrm{RZ}_{G, b}$ at $x$.

For an artinian local $\breve{\mathbb{Z}}_{p}$-algebra $R$ with residue field $\overline{\mathbb{F}}_{p}$, we define a deformation of $X_{x}$ over $R$ to be a $p$-divisible group $\mathscr{X}_{x}$ over $R$ with an isomorphism $\mathscr{X}_{x} \otimes_{R} \overline{\mathbb{F}}_{p} \cong X_{x}$. By Faltings in [Fal99], $\S 7$, there exists a formal scheme $\operatorname{Def}_{X_{x}, G}$ over $\operatorname{Spf}\left(\breve{\mathbb{Z}}_{p}\right)$ which classifies the deformations of $X_{x}$ with Tate tensors in the following sense: for a formally smooth $\breve{\mathbb{Z}}_{p}$-algebra of the form $R=\breve{\mathbb{Z}}_{p}\left[\left[u_{1}, \cdots, u_{N}\right]\right]$ or $R=\breve{\mathbb{Z}}_{p}\left[\left[u_{1}, \cdots, u_{N}\right]\right] /\left(p^{m}\right)$, $\operatorname{Def}_{X_{x}, G}(R)$ is the set of isomorphism classes of the pair $\left(\mathscr{X}_{x},\left(\mathbf{t}_{i}\right)\right)$ where

- $\mathscr{X}_{x}$ is a deformation of $X_{x}$ over $R$;

- $\left(\mathbf{t}_{i}\right)$ is a family of Frobenius-invariant tensors on $\mathbb{D}\left(\mathscr{X}_{x}\right)$ which lift the tensors $\left(t_{i}\right)$ and lie in the 0th filtration with respect to the Hodge filtration.

From this moduli description, we obtain a natural isomorphism

$$
\operatorname{Def}_{X_{x}, G} \simeq\left(\widehat{\mathrm{RZ}_{G, b}}\right)_{x}
$$

as explained in [Kim13, 4.8.

Now consider another unramified local Shimura datum $\left(G^{\prime},\left[b^{\prime}\right],\left\{\mu^{\prime}\right\}\right)$ and choose $b^{\prime} \in\left[b^{\prime}\right] \cap G\left(\breve{\mathbb{Z}}_{p}\right) \mu^{\prime}(p) G\left(\breve{\mathbb{Z}}_{p}\right)$ as in Proposition 3.2.1. For any point $x^{\prime} \in \mathrm{RZ}_{G^{\prime}, b^{\prime}}\left(\overline{\mathbb{F}}_{p}\right)$, the morphism in (1) of Proposition 3.2.1 induces an isomorphism

$$
\operatorname{Def}_{X_{x}, G} \times \operatorname{Def}_{X_{x^{\prime}}, G^{\prime}} \stackrel{\sim}{\longrightarrow} \operatorname{Def}_{X_{x} \times X_{x^{\prime}}, G \times G^{\prime}}
$$

defined by the product of deformations. For any homomorphism $f: G \longrightarrow G^{\prime}$ with $f(b)=b^{\prime}$, if we take $x^{\prime} \in \mathrm{RZ}_{G^{\prime}, b^{\prime}}\left(\overline{\mathbb{F}}_{p}\right)$ to be the image of $x$ under the morphism in $(2)$ of Proposition 3.2.1, we have an induced morphism

$$
\operatorname{Def}_{X_{x}, G} \longrightarrow \operatorname{Def}_{X_{x^{\prime}}, G^{\prime}}
$$

which is a closed embedding if $f$ is a closed embedding.

\subsection{Associated local Shimura varieties.}

3.3.1. Consider the algebraic group $J_{b}$ over $\mathbb{Q}_{p}$ defined in 2.2 .2 , Note that $J_{b}\left(\mathbb{Q}_{p}\right)$ can be identified with the group of quasi-isogenies $\gamma: X \longrightarrow X$ that preserve the tensors $\left(t_{i}\right)$. One can show that $\mathrm{RZ}_{G, b}$ carries a natural left $J_{b}\left(\mathbb{Q}_{p}\right)$-action defined by

$$
\gamma(\mathcal{X}, \iota)=\left(\mathcal{X}, \iota \circ \gamma^{-1}\right)
$$

for any $R \in \operatorname{Nilp}_{\breve{\mathbb{Z}}_{p}},(\mathcal{X}, \iota) \in \mathrm{RZ}_{G, b}(R)$ and $\gamma \in J_{b}\left(\mathbb{Q}_{p}\right)$ (see [Kim13], 7.2.). 
3.3.2. Let $E$ be the field of definition of the $G\left(\breve{\mathbb{Q}}_{p}\right)$-conjugacy class of $\mu$, and let $\mathscr{O}_{E}$ denote its ring of integers. Note that $E$ is a finite unramified extension of $\mathbb{Q}_{p}$ since $G_{\mathbb{Q}_{p}}$ is split over a finite unramified extension of $\mathbb{Q}_{p}$. Let $d$ be the degree of the extension, and write $\tau$ for the Frobenius automorphism of $\breve{\mathbb{Q}}_{p}$ relative to $E$.

For any formal scheme $S$ over $\operatorname{Spf}\left(\breve{\mathbb{Z}}_{p}\right)$, we write $S^{\tau}:=S \times_{\operatorname{Spf}\left(\breve{\mathbb{Z}}_{p}\right), \tau} \operatorname{Spf}\left(\breve{\mathbb{Z}}_{p}\right)$. By a Weil descent datum on $S$ over $\mathscr{O}_{E}$, we mean an isomorphism $S \stackrel{\sim}{\longrightarrow} S^{\tau}$. If $S \cong$ $S_{0} \times_{\operatorname{Spf}\left(\mathscr{O}_{E}\right)} \operatorname{Spf}\left(\breve{\mathbb{Z}}_{p}\right)$ for some formal scheme $S_{0}$ over $\operatorname{Spf}\left(\mathscr{O}_{E}\right)$, then there exists a natural Weil descent datum on $S$ over $\mathscr{O}_{E}$, called an effective Weil descent datum.

For any $R \in \operatorname{Nilp}_{\breve{\mathbb{Z}}_{p}}$, we define $R^{\tau}$ to be $R$ viewed as a $\breve{\mathbb{Z}}_{p}$-algebra via $\tau$. Note that we have a natural identification $\mathrm{RZ}_{b}^{\tau}(R)=\mathrm{RZ}_{b}\left(R^{\tau}\right)$. Following Rapoport and Zink in [RZ96], 3.48, we define a Weil descent datum $\Phi$ on $\mathrm{RZ}_{b}$ over $\mathscr{O}_{E}$ by sending $(\mathcal{X}, \iota) \in \mathrm{RZ}_{b}(R)$ with $R \in \operatorname{Nilp}_{\breve{Z}_{p}}$ to $\left(\mathcal{X}^{\Phi}, \iota^{\Phi}\right) \in \mathrm{RZ}_{b}\left(R^{\tau}\right)$ where

- $\mathcal{X}^{\Phi}$ is $\mathcal{X}$ viewed as a $p$-divisible group over $R^{\tau}$;

- $\iota^{\Phi}$ is the quasi-isogeny

$$
\iota^{\Phi}: X_{R^{\tau} / p}=\left(\tau^{*} X\right)_{R / p} \stackrel{\text { Frob }^{-d}}{\longrightarrow} X_{R / p} \stackrel{\iota}{\longrightarrow} \mathcal{X}_{R / p}=\mathcal{X}_{R / p}^{\Phi}
$$

where $\operatorname{Frob}^{d}: X \rightarrow \tau^{*} X$ is the relative $q$-Frobenius with $q=p^{d}$.

One can check that $\Phi$ restricts to a Weil descent datum $\Phi_{G}$ on $\mathrm{RZ}_{G, b}$ over $\mathscr{O}_{E}$ by looking at $\overline{\mathbb{F}}_{p}$-points and the formal completions thereof. The Weil descent datum $\Phi_{G}$ clearly commutes with the $J_{b}\left(\mathbb{Q}_{p}\right)$-action defined in 3.3.1.

3.3.3. Since $\mathrm{RZ}_{G, b}$ is locally formally of finite type over $\operatorname{Spf}\left(\breve{\mathbb{Z}}_{p}\right)$, it admits a rigid analytic generic fiber which we denote by $\mathrm{RZ}_{G, b}^{\text {rig }}$ (see $\left[\right.$ Ber96].). The $J_{b}\left(\mathbb{Q}_{p}\right)$-action and the Weil descent datum $\Phi_{G}$ on $\mathrm{RZ}_{G, b}$ induce an action of $J_{b}\left(\mathbb{Q}_{p}\right)$ on $\mathrm{RZ}_{G, b}^{\mathrm{rig}}$ and an Weil descent datum $\Phi_{G}: \mathrm{RZ} Z_{G, b}^{\text {rig }} \stackrel{\sim}{\longrightarrow}\left(\mathrm{RZ}_{G, b}^{\text {rig }}\right)^{\tau}$ over $E$.

Recall that we have a universal $p$-divisible group $\mathcal{X}_{G, b}$ over $\mathrm{RZ}_{G, b}$ and a family of universal tensors $\left(\mathbf{t}_{i}^{\text {univ }}\right)$ on $\mathbb{D}\left(\mathcal{X}_{G, b}\right)$. In addition, the family $\left(\mathbf{t}_{i}^{\text {univ }}\right)$ has a "étale realization" $\left(\mathbf{t}_{i \text {,ét }}^{\text {univ }}\right)$ on the Tate module $T_{p}\left(\mathcal{X}_{G, b}\right)$ (see [Kim13], Theorem 7.1.6.).

For any open compact subgroup $K_{p}$ of $G\left(\mathbb{Z}_{p}\right)$, we define the following rigid analytic étale cover of $\mathrm{RZ}_{G, b}^{\mathrm{rig}}$ :

$$
\mathrm{RZ}_{G, b}^{K_{p}}:=\mathbf{I s o m}_{\mathrm{RZ}}^{\mathrm{rig}}\left(\left[\Lambda,\left(s_{i}\right)\right],\left[T_{p}\left(\mathcal{X}_{G, b}\right),\left(\mathbf{t}_{i, \mathrm{e}}^{\mathrm{uni}}\right)\right]\right) / K_{p} .
$$

The $J_{b}\left(\mathbb{Q}_{p}\right)$-action and the Weil descent datum over $E$ on $\mathrm{RZ}_{G, b}^{\text {rig }}$ pull back to $\mathrm{RZ}_{G, b}^{K_{p}}$. As the level $K_{p}$ varies, these covers form a tower $\left\{\mathrm{RZ}_{G, b}^{K_{p}}\right\}$ with Galois group $G\left(\mathbb{Z}_{p}\right)$. We denote this tower by $\mathrm{RZ}_{G, b}^{\infty}$.

By [Kim13], Proposition 7.4.8, there exists a right $G\left(\mathbb{Q}_{p}\right)$-action on the tower $\mathrm{RZ}_{G, b}^{\infty}$ extending the Galois action of $G\left(\mathbb{Z}_{p}\right)$, which commutes with the natural $J_{b}\left(\mathbb{Q}_{p}\right)$-action and the Weil descent datum over $E$. In addition, there is a well-defined period map on $\mathrm{RZ} Z_{G, b}^{\text {rig }}$ as explained in [Kim13], 7.5. Hence the tower $\mathrm{RZ}_{G, b}^{\infty}$ is a local Shimura variety in the sense of Rapoport and Viehmann in [RV14, 5.1. 
3.3.4. We fix a prime $l \neq p$, and let $W_{E}$ denote the Weil group of $E$. For any level $K_{p} \subset G\left(\mathbb{Z}_{p}\right)$, we consider the cohomology groups

$$
H^{i}\left(\mathrm{RZ}_{G, b}^{K_{p}}\right)=H_{c}^{i}\left(\mathrm{RZ}_{G, b}^{K_{p}} \otimes_{\breve{\mathbb{Q}}_{p}} \mathbb{C}_{p}, \mathbb{Q}_{l}\left(\operatorname{dim} \mathrm{RZ}_{G, b}^{K_{p}}\right)\right)
$$

As the level $K_{p}$ varies, these cohomology groups form a tower $\left\{H^{i}\left(\mathrm{RZ}_{G, b}^{K_{p}}\right)\right\}$ for each $i$, endowed with a natural action of $G\left(\mathbb{Q}_{p}\right) \times W_{E} \times J_{b}\left(\mathbb{Q}_{p}\right)$.

Let $\rho$ be an admissible $l$-adic representation of $J_{b}\left(\mathbb{Q}_{p}\right)$. The groups

$$
H^{i, j}\left(\mathrm{RZ}_{G, b}^{\infty}\right)_{\rho}:=\underset{K_{p}}{\lim } \operatorname{Ext}_{J_{b}\left(\mathbb{Q}_{p}\right)}^{j}\left(H^{i}\left(\mathrm{RZ}_{G, b}^{K_{p}}\right), \rho\right)
$$

satisfy the following properties (see [RV14], Proposition 6.1 and [Man08], Theorem 8):

(1) The groups $H^{i, j}\left(\mathrm{RZ}_{G, b}^{\infty}\right)_{\rho}$ vanish for almost all $i, j$.

(2) There is a natural action of $G\left(\mathbb{Q}_{p}\right) \times W_{E}$ on each $H^{i, j}\left(\mathrm{RZ}_{G, b}^{\infty}\right)_{\rho}$.

(3) The representations $H^{i, j}\left(\mathrm{RZ}_{G, b}^{\infty}\right)_{\rho}$ are admissible.

Hence we can define a virtual representation of $G\left(\mathbb{Q}_{p}\right) \times W_{E}$

$$
H^{\bullet}\left(\mathrm{RZ}_{G, b}^{\infty}\right)_{\rho}:=\sum_{i, j \geq 0}(-1)^{i+j} H^{i, j}\left(\mathrm{RZ}_{G, b}^{\infty}\right)_{\rho}
$$

\section{Hodge-Newton Reducibility and Harris-Viehmann Conjecture}

\subsection{Harris-Viehmann conjecture: statement.}

4.1.1. Throughout this section, we fix a prime $p>2$ and an unramified local Shimura datum of Hodge type $(G,[b],\{\mu\})$. We also choose a faithful $G$-representation $\Lambda \in$ $\operatorname{Rep}_{\mathbb{Z}_{p}}(G)$ and a finite family of tensors $\left(s_{i}\right)$ on $\Lambda$ as in 3.1.3. In addition, we fix a maximal torus $T \subseteq G$ and a Borel subgroup $B \subseteq G$ containing $T$, both defined over $\mathbb{Z}_{p}$

Let $P$ be a proper standard parabolic subgroup of $G$ with Levi factor $L$ and unipotent radical $U$. For any element $b \in[b] \cap L\left(\breve{\mathbb{Q}}_{p}\right)$, we define $I_{b,\{\mu\}, L}$ to be the set of $L\left(\breve{\mathbb{Z}}_{p}\right)$ conjugacy classes of cocharacters of $L$ with a representative $\mu^{\prime}$ such that

(i) $\mu^{\prime} \in\{\mu\}_{G}$,

(ii) $[b]_{L} \cap L\left(\breve{\mathbb{Z}}_{p}\right) \mu^{\prime}(p) L\left(\breve{\mathbb{Z}}_{p}\right)$ is not empty.

Then $I_{b,\{\mu\}, L}$ is finite and nonempty (see [RV14], Lemma 8.1.).

Lemma 4.1.2. For any $\left\{\mu^{\prime}\right\}_{L} \in I_{b,\{\mu\}, L}$, the tuple $\left(L,[b]_{L},\left\{\mu^{\prime}\right\}_{L}\right)$ is an unramified local Shimura datum of Hodge type.

Proof. By construction, the tuple $\left(L,[b]_{L},\left\{\mu^{\prime}\right\}_{L}\right)$ satisfies the conditions (i) and (ii) of 3.1.1. Hence it remains to check the condition (iii) of 3.1.1. After taking $\sigma$-conjugate in $L\left(\breve{\mathbb{Q}}_{p}\right)$ if necessary, we may assume that $b \in L\left(\breve{\mathbb{Z}}_{p}\right) \mu^{\prime}(p) L\left(\breve{\mathbb{Z}}_{p}\right)$. Then we have $b \in G\left(\breve{\mathbb{Z}}_{p}\right) \mu(p) G\left(\breve{\mathbb{Z}}_{p}\right)$ since $\mu^{\prime} \in\{\mu\}$. Now we verify the condition (iii) with $b$ since $(G,[b],\{\mu\})$ is an unramified local Shimura datum of Hodge type.

We can now state the Harris-Viehmann conjecture in the setting of Rapoport-Zink spaces of Hodge type. 
Conjecture 4.1.3 ([RV14], Conjecture 8.4.). Choose an element $b \in[b] \cap G\left(\breve{\mathbb{Z}}_{p}\right) \mu(p) G\left(\breve{\mathbb{Z}}_{p}\right)$. Let $P$ be a parabolic subgroup of $G$ with Levi factor $L$ such that

(i) $[b] \cap L\left(\breve{\mathbb{Q}}_{p}\right)$ is not empty,

(ii) $J_{b}$ is an inner form of a Levi subgroup of $G$ contained in $L$.

Choose representatives $\mu_{1}, \mu_{2}, \cdots, \mu_{s}$ of the $L\left(\breve{\mathbb{Z}}_{p}\right)$-conjugacy classes of cocharacters in $I_{b,\{\mu\}, L}$, and also choose $b_{k} \in[b]_{L} \cap L\left(\breve{\mathbb{Z}}_{p}\right) \mu_{k}(p) L\left(\breve{\mathbb{Z}}_{p}\right)$ for each $k=1,2, \cdots, s$. Then for any admissible $\overline{\mathbb{Q}}_{l}$-representation $\rho$ of $J\left(\mathbb{Q}_{p}\right)$, we have an equality of virtual representations of $G\left(\mathbb{Q}_{p}\right) \times W_{E}$

$$
H^{\bullet}\left(R Z_{G, b}^{\infty}\right)_{\rho}=\bigoplus_{k=1}^{s} \operatorname{Ind} d_{P\left(\mathbb{Q}_{p}\right)}^{G\left(\mathbb{Q}_{p}\right)} H^{\bullet}\left(R Z_{L, b_{k}}^{\infty}\right)_{\rho}
$$

In particular, the virtual representation $H^{\bullet}\left(R Z_{G, b}^{\infty}\right)_{\rho}$ contains no supercuspidal representations of $G\left(\mathbb{Q}_{p}\right)$.

Here we consider the groups $H^{\bullet}\left(\mathrm{RZ}_{L, b_{k}}^{\infty}\right)_{\rho}$ as a virtual representation of $P\left(\mathbb{Q}_{p}\right) \times W_{E}$ by letting the unipotent radical of $P\left(\mathbb{Q}_{p}\right)$ act trivially. Note that the choice of $b_{k}$ 's (or $\mu_{k}$ 's) is unimportant since the isomorphism class of the spaces $\mathrm{RZ}_{L, b_{k}}^{\infty}$ only depend on the tuples $\left(L,[b]_{L},\left\{\mu_{k}\right\}_{L}\right)$.

4.1.4. We will prove Conjecture 4.1.3 under the assumption that the datum $(G,[b],\{\mu\})$ is Hodge-Newton reducible (with respect to $P$ and $L$ ). By definition, this means that there exist $\mu \in\{\mu\}$ and $b \in[b] \cap L\left(\breve{\mathbb{Q}}_{p}\right)$ with the following properties:

(i) the cocharacter $\mu$ factors through $L$,

(ii) $[b]_{L} \cap L\left(\breve{\mathbb{Z}}_{p}\right) \mu(p) L\left(\breve{\mathbb{Z}}_{p}\right)$ is not empty,

(iii) in the action of $\mu$ and $\nu_{b}$ on $\operatorname{Lie}(U) \otimes_{\mathbb{Q}_{p}} \breve{\mathbb{Q}}_{p}$, only non-negative characters occur.

Here $\nu_{b}$ denote the Newton cocharacter associated to $b$ (see [Ko85], $\S 4$ or [RR96], $\S 1$ for definition.). Note that the properties (i) and (ii) together imply that $\{\mu\}_{L} \in I_{b,\{\mu\}, L}$.

The notion of Hodge-Newton reducibility first appeared in [Ka79, where Katz considered $p$-divisible groups (and $F$-crystals) with the property that the Hodge polygon passes through a break point of the Newton polygon. For $G=G L_{n}$, our notion of Hodge-Newton reducibility is equivalent to the notion considered by Katz. More precisely, if $X$ is a $p$-divisible group over $\overline{\mathbb{F}}_{p}$ that arises from the datum $(G,[b],\{\mu\})$ with a choice of $b \in[b] \cap G\left(\breve{\mathbb{Z}}_{p}\right) \mu(p) G\left(\breve{\mathbb{Z}}_{p}\right)$, the datum $(G,[b],\{\mu\})$ is Hodge-Newton reducible (with respect to some parabolic subgroup and its Levi factor) if and only if the Hodge polygon of $X$ passes through a break point of the Newton polygon of $X$. See [RV14], Remark 4.25 for more details.

We want to interpret the statement of Conjecture 4.1.3 under our assumption. Let us choose $\mu \in\{\mu\}$ and $b \in[b] \cap L\left(\breve{\mathbb{Q}}_{p}\right)$ with the properties (i), (ii), and (iii) above. After replacing by a $\sigma$-conjugate if necessary, we may assume that $b \in L\left(\breve{\mathbb{Z}}_{p}\right) \mu(p) L\left(\breve{\mathbb{Z}}_{p}\right)$. Then $b$ and $L$ clearly satisfy the condition (i) of Conjecture 4.1.3. One can also check that $b$ and $L$ satisfy the condition (ii)] of Conjecture 4.1.3 (see [RV14], Remark 8.9.). Moreover, under our assumption the set $I_{b,\{\mu\}, L}$ consists of a single element, namely $\{\mu\}_{L}$ (see [RV14], Theorem 8.8.). 
Hence we may state our main theorem as follows:

Theorem 4.1.5. Assume that $(G,[b],\{\mu\})$ is Hodge-Newton reducible with respect to a standard parabolic subgroup $P$ with Levi factor $L$. Choose $\mu \in\{\mu\}$ and $b \in$ $L\left(\breve{\mathbb{Z}}_{p}\right) \mu(p) L\left(\breve{\mathbb{Z}}_{p}\right)$ with the properties (i), (ii) and (iii) of 4.1.4. Then for any admissible $\overline{\mathbb{Q}}_{l}$-representation $\rho$ of $J\left(\mathbb{Q}_{p}\right)$, we have an equality of virtual representations of $G\left(\mathbb{Q}_{p}\right) \times W_{E}$

$$
H^{\bullet}\left(R Z_{G, b}^{\infty}\right)_{\rho}=\operatorname{Ind} d_{P\left(\mathbb{Q}_{p}\right)}^{G\left(\mathbb{Q}_{p}\right)} H^{\bullet}\left(R Z_{L, b}^{\infty}\right)_{\rho} .
$$

In particular, the virtual representation $H^{\bullet}\left(R Z_{G, b}^{\infty}\right)_{\rho}$ contains no supercuspidal representations of $G\left(\mathbb{Q}_{p}\right)$.

\subsection{Rigid analytic tower associated to the parabolic subgroup.}

For our proof of Theorem 4.1.5, we construct an intermediate tower of rigid analytic spaces associated to the parabolic subgroup $P$.

4.2.1. For the rest of this section, we will always keep the assumption and the notations in the statement of Theorem 4.1.5. In addition, we write $\underline{X}=\left(X,\left(t_{i}\right)\right)$ for the $p$-divisible group with $G$-structure that arises from the datum $(G,[b],\{\mu\})$ with the choice $b \in[b]$.

By Hong16, Lemma 3.1.4, we can choose a group $\widetilde{G}$ of EL type with the following properties:

(i) the embedding $G \hookrightarrow \operatorname{GL}(\Lambda)$ factors through $\widetilde{G}$,

(ii) the datum $(\widetilde{G},[b],\{\mu\})$ is Hodge-Newton reducible with respect to a proper parabolic subgroup $\widetilde{P}$ of $\widetilde{G}$ and its Levi factor $\widetilde{L}$ such that $P=\widetilde{P} \cap G$ and $L=\widetilde{L} \cap G$.

In general, the group $\widetilde{G}$ is of the form

$$
\widetilde{G}=\operatorname{Res}_{\mathscr{O}_{1} \mid \mathbb{Z}_{p}} \mathrm{GL}_{n_{1}} \times \operatorname{Res}_{\mathscr{O}_{2} \mid \mathbb{Z}_{p}} \mathrm{GL}_{n_{2}} \times \operatorname{Res}_{\mathscr{O}_{f} \mid \mathbb{Z}_{p}} \mathrm{GL}_{n_{f}}
$$

where each $\mathscr{O}_{j}$ is the ring of integers for some finite unramified extension of $\mathbb{Q}_{p}$. However, in light of functorial properties in Proposition 3.2.1, we may assume for simplicity that

$$
\widetilde{G}=\operatorname{Res}_{\mathscr{O} \mid \mathbb{Z}_{p}} \mathrm{GL}_{n}
$$

where $\mathscr{O}$ is the integer ring of some finite unramified extension of $\mathbb{Q}_{p}$. Then the Levi subgroup $\widetilde{L}$ takes the form

$$
\widetilde{L}=\operatorname{Res}_{\mathscr{O} \mid \mathbb{Z}_{p}} \mathrm{GL}_{m_{1}} \times \operatorname{Res}_{\mathscr{O} \mid \mathbb{Z}_{p}} \mathrm{GL}_{m_{2}} \times \cdots \times \operatorname{Res}_{\mathscr{O} \mid \mathbb{Z}_{p}} \mathrm{GL}_{m_{r}}
$$

For each $j=1,2, \cdots, r$, we define the following data:

- $\widetilde{L}_{j}$ is the $j$-th factor in the decomposition (4.2.1.1),

- $L_{j}$ is the image of $L$ under the projection $\widetilde{L} \rightarrow \widetilde{L}_{j}$,

- $b_{j}$ is the image of $b$ under the projection $L \rightarrow L_{j}$,

- $\mu_{j}$ is the cocharacter of $L_{j}$ induced from $\mu$ via the projection $L \rightarrow L_{j}$. 
4.2.2. For any $x \in \mathrm{RZ}_{G, b}\left(\overline{\mathbb{F}}_{p}\right)$, we write $\left(X_{x},\left(t_{x, i}\right), \iota_{x}\right)$ for the corresponding tuple under the moduli description of $\mathrm{RZ}_{G, b}\left(\overline{\mathbb{F}}_{p}\right)$ described in 3.1 .6 , and $\underline{X}_{x}:=\left(X_{x},\left(t_{x, i}\right)\right)$ for the associated $p$-divisible group with $G$-structure. Then we have the following facts from Hong16, §3.2:

(1) The tuples $\left(\widetilde{L}_{j},\left[b_{j}\right],\left\{\mu_{j}\right\}\right)$ and $\left(L_{j},\left[b_{j}\right],\left\{\mu_{j}\right\}\right)$ are unramified local Shimura data of Hodge type for each $j=1,2, \cdots, s$.

(2) There is a natural map of the affine Deligne-Lusztig sets

$$
X_{\{\mu\}}^{G}([b]) \stackrel{\sim}{\longrightarrow} X_{\{\mu\}}^{L}([b]) \longleftrightarrow X_{\left\{\mu_{1}\right\}}^{L_{1}}\left(\left[b_{1}\right]\right) \times \cdots \times X_{\left\{\mu_{r}\right\}}^{L_{r}}\left(\left[b_{r}\right]\right)
$$

which induces a natural map

$$
\mathrm{RZ}_{G, b}\left(\overline{\mathbb{F}}_{p}\right) \stackrel{\sim}{\longrightarrow} \mathrm{RZ}_{L, b}\left(\overline{\mathbb{F}}_{p}\right) \hookrightarrow \mathrm{RZ}_{L_{1}, b_{1}}\left(\overline{\mathbb{F}}_{p}\right) \times \cdots \times \mathrm{RZ}_{L_{r}, b_{r}}\left(\overline{\mathbb{F}}_{p}\right)
$$

via the natural bijections between the affine Deligne-Lusztig sets and the set of $\overline{\mathbb{F}}_{p}$-valued points of the Rapoport-Zink spaces (see 3.2.2.).

(3) The second map in (2) induces a decomposition

$$
\underline{X}_{x}=\underline{X}_{x_{1}} \times \underline{X}_{x_{2}} \times \cdots \times \underline{X}_{x_{r}}
$$

where $\underline{X}_{x_{j}}$ is the $p$-divisible group with $L_{j}$-structure corresponding to the image of $x$ in $\mathrm{RZ}_{L_{j}, b_{j}}\left(\overline{\mathbb{F}}_{p}\right)$.

(4) If we set $X_{x}^{(j)}:=X_{x_{j}} \times X_{x_{j+1}} \times \cdots \times X_{x_{r}}$ for each $j=1,2, \cdots, r$, the decomposition in (3) induces a filtration

$$
0 \subset X_{x}^{(r)} \subset X_{x}^{(r-1)} \subset \cdots \subset X_{x}^{(1)}=X_{x}
$$

such that each quotient $X_{x}^{(j)} / X_{x}^{(j+1)} \simeq X_{x_{j}}$ carries $L_{j}$-structure that arises from the datum $\left(L_{j},\left[b_{j}\right],\left\{\mu_{j}\right\}\right)$.

(5) Given a formally smooth $\breve{\mathbb{Z}}_{p}$-algebra of the form $R=\breve{\mathbb{Z}}_{p}\left[\left[u_{1}, \cdots, u_{N}\right]\right]$ or $R=$ $\breve{\mathbb{Z}}_{p}\left[\left[u_{1}, \cdots, u_{N}\right]\right] /\left(p^{m}\right)$ and a deformation $\underline{\mathscr{X}}_{x}=\left(\mathscr{X}_{x},\left(\mathbf{t}_{i}\right)\right) \in \operatorname{Def}_{X_{x}, G}(R)$ with an isomorphism $\alpha: \mathscr{X}_{x} \otimes_{R} \overline{\mathbb{F}}_{p} \cong X_{x}$, there exists a unique filtration of $\mathscr{X}$

$$
0 \subset \mathscr{X}_{x}^{(r)} \subset \mathscr{X}_{x}^{(r-1)} \subset \cdots \subset \mathscr{X}_{x}^{(1)}=\mathscr{X}_{x}
$$

which lifts the filtration in (4) in the following sense: for each $j=1,2, \cdots, r$, the isomorphism $\alpha$ induces isomorphisms $\alpha^{(j)}: \mathscr{X}_{x}^{(j)} \otimes_{R} \overline{\mathbb{F}}_{p} \cong X_{x}^{(j)}$ and $\alpha_{j}$ : $\left(\mathscr{X}_{x}^{(j)} / \mathscr{X}_{x}^{(j+1)}\right) \otimes_{R} \overline{\mathbb{F}}_{p} \cong X_{x_{j}}$ such that $\left(\mathscr{X}_{x}^{(j)} / \mathscr{X}_{x}^{(j+1)},\left(\mathbf{t}_{i}^{(j)}\right)\right) \in \operatorname{Def}_{X_{x_{j}}, L_{j}}(R)$ for some family of tensors $\left(\mathbf{t}_{i}^{(j)}\right)$ on $\mathbb{D}\left(\mathscr{X}_{x}^{(j)} / \mathscr{X}_{x}^{(j+1)}\right)$.

We refer to the decomposition in (3) and the filtration in (4) respectively as the HodgeNewton decomposition and the Hodge-Newton filtration of $\underline{X}_{x}$ (with respect to $P$ and $L)$. If we take $x$ such that $X_{x}=X,\left(t_{x, i}\right)=\left(t_{i}\right)$ and $\iota_{x}$ is the identity map on $X$, we obtain the Hodge-Newton decomposition of $\underline{X}$

$$
\underline{X}=\underline{X}_{1} \times \underline{X}_{2} \times \cdots \times \underline{X}_{r}
$$

and the corresponding Hodge-Newton filtration of $\underline{X}$

$$
0 \subset X^{(r)} \subset X^{(r-1)} \subset \cdots \subset X^{(1)}=X
$$

where each quotient $X^{(j)} / X^{(j+1)} \simeq X_{j}$ carries $L_{j}$-structure that arises from the datum $\left(L_{j},\left[b_{j}\right],\left\{\mu_{j}\right\}\right)$ with the choice $b_{j} \in\left[b_{j}\right]$. 
4.2.3. Following Mantovan in [Man08], Definition 9, we define a set-valued functor $\mathrm{RZ}_{\widetilde{P}, b}$ on $\operatorname{Nilp}_{\breve{\mathbb{Z}}_{p}}$ as follows: for any $R \in \mathrm{Nilp}_{\breve{\mathbb{Z}}_{p}}$, we set $\mathrm{RZ}_{\widetilde{P}, b}(R)$ to be the set of isomorphism classes of triples $(\mathcal{X}, \mathcal{X} \bullet, \iota)$ where

- $\mathcal{X}$ is a $p$-divisible group over $R$ with an action of $\mathscr{O}$ (see Example 3.1.8);

- $\mathcal{X}^{\bullet}$ is a filtration of $p$-divisible groups over $R$

$$
0 \subset \mathcal{X}^{(r)} \subset \mathcal{X}^{(r-1)} \subset \cdots \subset \mathcal{X}^{(1)}=\mathcal{X}
$$

which is preserved by the action of $\mathscr{O}$ such that the quotients $\mathcal{X}^{(j)} / \mathcal{X}^{(j+1)}$ are $p$-divisible groups (with the induced action of $\mathscr{O}$ );

- $\iota: X_{R / p} \rightarrow \mathcal{X}_{R / p}$ is a quasi-isogeny which is compatible with the action of $\mathscr{O}$ and induces quasi-isogenies $\iota^{(j)}: X_{R / p}^{(j)} \longrightarrow \mathcal{X}_{R / p}^{(j)}$ for $j=1,2, \cdots, r$,

such that for all $a \in \mathscr{O}$ and $j=1,2, \cdots, r$,

$$
\operatorname{det}_{R}\left(a, \operatorname{Lie}\left(\mathcal{X}^{(j)}\right)\right)=\operatorname{det}\left(a, \operatorname{Fil}^{0}\left(X^{(j)}\right)_{\breve{Q}_{p}}\right) \text {. }
$$

Mantovan in [Man08], Proposition 11 proved that the functor $\mathrm{RZ}_{\widetilde{P}, b}$ is represented by a formal scheme which is formally smooth and locally formally of finite type over $\breve{\mathbb{Z}}_{p}$. We write $\mathrm{RZ}_{\widetilde{P}, b}$ also for this representing formal scheme, and $\mathrm{RZ}_{\widetilde{P}, b}^{\text {rig }}$ for its rigid analytic generic fiber. In addition, we write $\mathcal{X}_{\widetilde{P}, b}$ and $\mathcal{X}_{\widetilde{P}, b}^{\bullet}$ respectively for the universal filtered $p$-divisible group over $\mathrm{RZ}_{\widetilde{P}, b}$ and the associated "universal filtration".

Remark. As in Man08, Definition 10, we can also define a tower of étale covers $\mathrm{RZ}_{\widetilde{P}, b}^{\infty}=\left\{\mathrm{RZ}_{X, \widetilde{P}}^{\widetilde{K}_{p}}\right\}$ over $\mathrm{RZ}_{\widetilde{P}, b}^{\text {rig }}$ with a natural action of $\widetilde{P}\left(\mathbb{Q}_{p}\right) \times J_{b}\left(\mathbb{Q}_{p}\right)$ and a Weil descent datum over $E$, where $\widetilde{K}_{p}^{\prime}$ runs over open and compact subgroups of $\widetilde{P}\left(\mathbb{Z}_{p}\right)$.

4.2.4. By the functoriality of Rapoport-Zink spaces described in Proposition 3.2.1, the embedding $G \longleftrightarrow \widetilde{G}$ induces a closed embedding

$$
\mathrm{RZ}_{G, b} \longleftrightarrow \mathrm{RZ}_{\widetilde{G}, b} .
$$

In addition, we have a natural map

$$
\widetilde{\pi}_{2}: \mathrm{RZ}_{\widetilde{P}, b} \longrightarrow \mathrm{RZ}_{\widetilde{G}, b}
$$

defined by $(\mathcal{X}, \mathcal{X} \bullet, \iota) \mapsto(\mathcal{X}, \iota)$ on the points. We define $\mathrm{RZ}_{P, b}:=\mathrm{RZ}_{\widetilde{P}, b} \times_{\mathrm{RZ}_{\widetilde{G}, b}} \mathrm{RZ}_{G, b}$. Then we have the following Cartesian diagram:

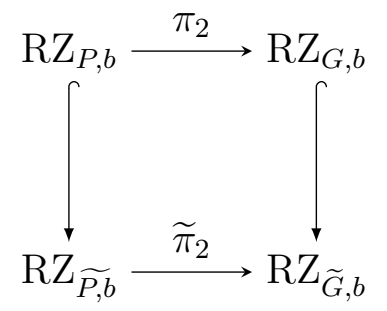

Moreover, $\pi_{2}$ is a local isomorphism which gives an isomorphism on the rigid analytic generic fiber since $\widetilde{\pi}_{2}$ has the same properties (see [Man08], Theorem 36 and [Sh13], Proposition 6.3.). 
We want to describe the universal property of the closed embedding $\mathrm{RZ}_{P, b} \longleftrightarrow \mathrm{RZ}_{\widetilde{P}, b}$ in an analogous way to the universal property of $\mathrm{RZ}_{G, b} \subset \mathrm{RZ}_{b}$ described in 3.1.5. For this, we choose a decomposition of $\Lambda$

$$
\Lambda=\Lambda_{1} \oplus \Lambda_{2} \oplus \cdots \oplus \Lambda_{r}
$$

corresponding to the decomposition of $\widetilde{L}$ in (4.2.1.1). We set $\Lambda^{(j)}=\Lambda_{1} \oplus \cdots \oplus \Lambda_{j}$ for $j=1,2, \cdots, r$, and denote by $\Lambda^{\bullet}$ the filtration

$$
0 \subset \Lambda^{(1)} \subset \cdots \subset \Lambda^{(r)}=\Lambda
$$

Then for any $\mathbb{Z}_{p}$-algebra $R$ we have

$$
P(R)=\left\{g \in G(R): g\left(\Lambda_{R}^{\bullet}\right)=\Lambda_{R}^{\bullet} .\right.
$$

Now consider a morphism $f: \operatorname{Spf}(R) \rightarrow \mathrm{RZ}_{\widetilde{P}, b}$ for some $R \in \operatorname{Nilp}_{\breve{\mathbb{Z}}_{p}}$. Let $\left(\mathcal{X}, \mathcal{X}^{\bullet}\right)$ be a $p$-divisible group over $\operatorname{Spec}(R)$ with a filtration which pulls back to $\left(f^{*} \mathcal{X}_{\widetilde{P}, b}, f^{*} \mathcal{X}_{\widetilde{P}, b}\right)$ over Spf $(R)$. We denote by $\mathbb{D}\left(\mathcal{X}^{\bullet}\right)$ the filtration of Dieudonné modules

$$
0=\mathbb{D}\left(\mathcal{X} / \mathcal{X}^{(1)}\right) \subset \mathbb{D}\left(\mathcal{X} / \mathcal{X}^{(2)}\right) \subset \cdots \subset \mathbb{D}\left(\mathcal{X} / \mathcal{X}^{(r)}\right) \subset \mathbb{D}(\mathcal{X})
$$

induced by $\mathcal{X} \bullet$ via (contravariant) Dieudonne theory. We choose tensors $\left(\hat{t}_{i}\right)$ on $\mathbb{D}(\mathcal{X})[1 / p]$ as in 3.1.5. Then $f$ factors through $\mathrm{RZ}_{P, b}$ if and only if $\widetilde{\pi}_{2} \circ f$ factors through $\mathrm{RZ}_{G, b} \longleftrightarrow$ $\mathrm{RZ}_{\widetilde{G}, b}$, which is equivalent to existence of a (unique) family of tensors $\left(\mathbf{t}_{i}\right)$ on $\mathbb{D}(\mathcal{X})$ such that

(i) for some ideal of definition $J$ of $R$ containing $p$, the pull-back of $\left(\mathbf{t}_{i}\right)$ over $R / J$ agrees with the pull-back of $\left(\hat{t}_{i}\right)$ over $R / J$,

(ii) for a $p$-adic lift $\mathcal{R}$ of $R$ which is formally smooth over $\breve{\mathbb{Z}}_{p}$, the $\mathcal{R}$-scheme

$$
\mathcal{P}_{\mathcal{R}}:=\operatorname{Isom}_{\mathcal{R}}\left(\left[\mathbb{D}(\mathcal{X})_{\mathcal{R}},\left(\mathbf{t}_{i}\right)_{\mathcal{R}}\right],\left[\Lambda^{*} \otimes_{\mathbb{Z}_{p}} \mathcal{R},\left(s_{i} \otimes 1\right)\right]\right)
$$

defined in 3.1 .5 is a $G$-torsor, and consequently the $\mathcal{R}$-scheme

$$
\mathcal{P}_{\mathcal{R}}^{\prime}:=\operatorname{Isom}_{\mathcal{R}}\left(\left[\mathbb{D}\left(\mathcal{X}^{\bullet}\right)_{\mathcal{R}},\left(\mathbf{t}_{i}\right)_{\mathcal{R}}\right],\left[\left(\Lambda^{\bullet}\right)^{*} \otimes_{\mathbb{Z}_{p}} \mathcal{R},\left(s_{i} \otimes 1\right)\right]\right)
$$

is a $P$-torsor,

(iii) the Hodge filtration of $\mathcal{X}$ is a $\{\mu\}$-filtration with respect to $\left(\mathbf{t}_{i}\right)$.

Here the scheme $\mathcal{P}_{\mathcal{R}}^{\prime}$ in (ii) classifies the isomorphisms $\mathbb{D}(\mathcal{X})_{\mathcal{R}} \cong \Lambda_{\mathcal{R}}^{*}$ which map the tensors $\left(\mathbf{t}_{i}\right)$ to $\left(s_{i} \otimes 1\right)$ and the filtration $\mathbb{D}\left(\mathcal{X}^{\bullet}\right)_{R}$ to $\left(\Lambda^{\bullet}\right)^{*} \otimes_{\mathbb{Z}_{p}} \mathcal{R}$.

We obtain the "universal $p$-divisible group" $\mathcal{X}_{P, b}$ over $\mathrm{RZ}_{P, b}$ with the associated "universal filtration" $\mathcal{X}_{P, b}^{\bullet}$ by taking the pull-back of $\mathcal{X}_{\widetilde{P}, b}$ and $\mathcal{X}_{\widetilde{P}, b}^{\bullet}$ over $\mathrm{RZ}_{P, b}$. We also obtain a family of "universal tensors" $\left(\mathbf{t}_{i}^{\text {univ }, P}\right)$ on $\mathbb{D}\left(\mathcal{X}_{P, b}\right)$ by applying the universal property to an open affine covering of $\mathrm{RZ}_{P, b}$. Moreover, this family has a "étale realization" $\left(\mathbf{t}_{i, \text { út }}^{\text {univ } P}\right)$ on the Tate module $T_{p}\left(\mathcal{X}_{P, b}\right)$ (see [Kim13], Theorem 7.1.6.). 
4.2.5. The formal scheme $\mathrm{RZ}_{P, b}$ is formally smooth and locally formally of finite type over $\breve{\mathbb{Z}}_{p}$ by construction. Hence it admits a rigid analytic generic fiber which we denote by $\mathrm{RZ}_{\widetilde{P}, b}^{\text {rig }}$. Moreover, since $\pi_{2}$ gives an isomorphism on the rigid analytic generic fiber, we have a $J_{b}\left(\mathbb{Q}_{p}\right)$-action and a Weil descent datum over $E$ on $\mathrm{RZ}_{P, b}^{\text {rig }}$ induced by the corresponding structures on $\mathrm{RZ}_{G, b}^{\text {rig }}$.

For any open compact subgroup $K_{p}{ }^{\prime}$ of $P\left(\mathbb{Z}_{p}\right)$, we define the following rigid analytic étale cover of $\mathrm{RZ} \mathrm{r}_{P, b}^{\mathrm{rig}}$ :

$$
\mathrm{RZ}_{P, b}^{K_{p}{ }^{\prime}}:=\mathbf{I s o m}_{\mathrm{RZ}} Z_{P, b}^{\text {rig }}\left(\left[\Lambda^{\bullet},\left(s_{i}\right)\right],\left[T_{p}\left(\mathcal{X}_{P, b}^{\bullet}\right),\left(\mathbf{t}_{i, \text { ét }}^{\text {univ }, P}\right)\right]\right) / K_{p}{ }^{\prime} .
$$

The $J_{b}\left(\mathbb{Q}_{p}\right)$-action and the Weil descent datum over $E$ on $\mathrm{RZ}_{P, b}^{\mathrm{rig}}$ pull back to $\mathrm{RZ} Z_{P, b}^{K_{p}{ }^{\prime}}$. We denote by $\mathrm{RZ}_{P, b}^{\infty}:=\left\{\mathrm{RZ}_{P, b}^{K_{p}{ }^{\prime}}\right\}$ the tower of these covers with Galois group $P\left(\mathbb{Z}_{p}\right)$. The Galois action on this tower gives rise to a natural $P\left(\mathbb{Q}_{p}\right)$-action which commutes with the $J_{b}\left(\mathbb{Q}_{p}\right)$-action and the Weil descent datum over $E$ (cf. [Kim13, Proposition 7.4.8.). Hence the cohomology groups

$$
H^{i}\left(\mathrm{RZ}_{P, b}^{K_{p}{ }^{\prime}}\right)=H_{c}^{i}\left(\mathrm{RZ}_{P, b}^{K_{p}} \otimes_{\breve{Q}_{p}} \mathbb{C}_{p}, \mathbb{Q}_{l}\left(\operatorname{dim} \mathrm{RZ}_{P, b}^{K_{p}{ }^{\prime}}\right)\right)
$$

form a tower $\left\{H^{i}\left(\mathrm{RZ}_{P, b}^{K_{p}{ }^{\prime}}\right)\right\}$ for each $i$, which are endowed with a natural action of $P\left(\mathbb{Q}_{p}\right) \times W_{E} \times J_{b}\left(\mathbb{Q}_{p}\right)$. Moreover, for any admissible $l$-adic representation $\rho$ of $J_{b}\left(\mathbb{Q}_{p}\right)$, the groups

$$
H^{i, j}\left(\mathrm{RZ}_{P, b}^{\infty}\right)_{\rho}:=\underset{\mathrm{K}_{p}^{\prime}}{\lim _{\overrightarrow{1}}} \operatorname{Ext}_{J_{b}\left(\mathbb{Q}_{p}\right)}^{j}\left(H^{i}\left(\mathrm{RZ}_{P, b}^{K_{p}{ }^{\prime}}\right), \rho\right)
$$

satisfy the following properties (cf. 3.3.4):

(1) The groups $H^{i, j}\left(\mathrm{RZ}_{P, b}^{\infty}\right)_{\rho}$ vanish for almost all $i, j$.

(2) There is a natural action of $P\left(\mathbb{Q}_{p}\right) \times W_{E}$ on each $H^{i, j}\left(\mathrm{RZ}_{P, b}^{\infty}\right)_{\rho}$.

(3) The representations $H^{i, j}\left(\mathrm{RZ}_{P, b}^{\infty}\right)_{\rho}$ are admissible.

We can thus define a virtual representation of $P\left(\mathbb{Q}_{p}\right) \times W_{E}$

$$
H^{\bullet}\left(\mathrm{RZ}_{P, b}^{\infty}\right)_{\rho}:=\sum_{i, j \geq 0}(-1)^{i+j} H^{i, j}\left(\mathrm{RZ}_{P, b}^{\infty}\right)_{\rho} .
$$

Remark. Alternatively, we can obtain the tower $\mathrm{RZ}_{P, b}^{\infty}$ as the pull-back of the tower $\mathrm{RZ}_{\widetilde{P}, b}^{\infty}$ over $\mathrm{RZ}_{P, b}^{\mathrm{rig}}$.

\subsection{Harris-Viehmann conjecture: proof.}

We finally present our proof of Theorem 4.1.5. We retain all the notations from 4.2.

Lemma 4.3.1. There exists a diagram

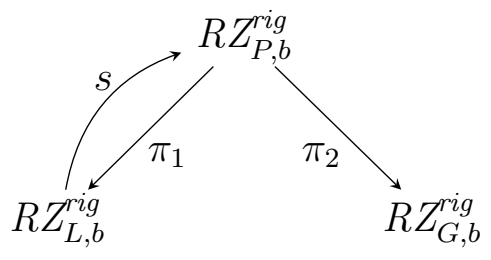


such that

(1) $s$ is a closed immersion,

(2) $\pi_{1}$ is a fibration in balls,

(3) $\pi_{2}$ is an isomorphism.

Proof. For notational simplicity, we assume that $r=2$, i.e., the decomposition of $\widetilde{L}$ in (4.2.1.1) has two factors. Our argument will naturally extend to the general case.

Note that we have already constructed $\pi_{2}$ and proved $(3)$ in 4.2 .4 .

Let us now prove $(1)$. From the decomposition $\widetilde{L}=\widetilde{L}_{1} \times \widetilde{L}_{2}$ we obtain a natural isomorphism $\mathrm{RZ}_{\widetilde{L}, b} \simeq \mathrm{RZ}_{\widetilde{L}_{1}, b_{1}} \times \mathrm{RZ}_{\widetilde{L}_{2}, b_{2}}$ by Proposition 3.2.1. Consider the map

$$
\widetilde{s}: \mathrm{RZ}_{\widetilde{L}, b} \simeq \mathrm{RZ}_{\widetilde{L}_{1}, b_{1}} \times \mathrm{RZ}_{\widetilde{L}_{2}, b_{2}} \longrightarrow \mathrm{RZ}_{\widetilde{P}_{, b}}
$$

where the second arrow is defined by $\left(\mathcal{X}_{1}, \iota_{1}, \mathcal{X}_{2}, \iota_{2}\right) \mapsto\left(\mathcal{X}_{1} \times \mathcal{X}_{2}, 0 \subset \mathcal{X}_{2} \subset \mathcal{X}_{1} \times \mathcal{X}_{2}, \iota_{1} \times \iota_{2}\right)$ on the points. Then $\widetilde{s}$ gives a closed immersion on the rigid analytic generic fibers by Man08, Proposition 14. We define $s$ to be the restriction of $\widetilde{s}$ on $\mathrm{RZ}_{L, b}$. Since $s$ also gives a closed immersion on the rigid analytic generic fibers by construction, it suffices to show that $s$ factors through the embedding $\mathrm{RZ}_{P, b} \longleftrightarrow \mathrm{RZ}_{\widetilde{P}, b}$, which amounts to proving that $\widetilde{\pi}_{2} \circ s$ factors through $\mathrm{RZ}_{G, b}$. In fact, $\widetilde{\pi}_{2} \circ s$ is the natural closed embedding $\mathrm{RZ}_{L, b} \hookrightarrow \mathrm{RZ}_{\widetilde{G}, b}$ which is functorially induced by the embedding $L \hookrightarrow \widetilde{G}$ in the sense of Proposition 3.2.1. Hence $\tilde{\pi}_{2} \circ s$ factors through $\mathrm{RZ}_{G, b}$ as the embedding $L \hookrightarrow \widetilde{G}$ factors through $G$.

It remains to prove (2). Note that we have a natural embedding

$$
\mathrm{RZ}_{L, b} \longleftrightarrow \mathrm{RZ}_{\widetilde{L}, b}
$$

which is functorially induced by the embedding $L \hookrightarrow \widetilde{L}$ in the sense of Proposition 3.2.1. Consider the map

$$
\widetilde{\pi}_{1}: \mathrm{RZ}_{\widetilde{P}, b} \longrightarrow \mathrm{RZ}_{\widetilde{L}_{1}, b_{1}} \times \mathrm{RZ}_{\widetilde{L}_{2}, b_{2}} \stackrel{\sim}{\longrightarrow} \mathrm{RZ}_{\widetilde{L}, b}
$$

defined by $\left(\mathcal{X}, \mathcal{X}^{\bullet}, \iota\right) \mapsto\left(\mathcal{X} / \mathcal{X}^{(2)}, \iota / \iota^{(2)}, \mathcal{X}^{(2)}, \iota^{(2)}\right) \mapsto\left(\left(\mathcal{X} / \mathcal{X}^{(2)}\right) \times \mathcal{X}^{(2)},\left(\iota / \iota^{(2)}\right) \times \iota^{(2)}\right)$ on the points, where $\iota / \iota^{(2)}:\left(X_{1}\right)_{R / p}=\left(X / X^{(2)}\right)_{R / p} \longrightarrow\left(\mathcal{X} / \mathcal{X}^{(2)}\right)_{R / p}$ is a quasi-isogeny induced by $\iota$ and $\iota^{(2)}$. We define $\pi_{1}$ be the restriction of $\widetilde{\pi}_{1}$ on $\mathrm{RZ}_{P, b}$.

We claim that $\pi_{1}$ factor through the embedding $\mathrm{RZ}_{L, b} \longleftrightarrow \mathrm{RZ}_{\widetilde{L}, b}$. It suffices to show that (locally) the map $\pi_{2}^{-1} \circ \pi_{1}$ factors through $\mathrm{RZ}_{L, b} \longleftrightarrow \mathrm{RZ}_{\widetilde{L}, b}$. We only need to check this on the set of $\overline{\mathbb{F}}_{p}$-points and the completions thereof. On the set of $\overline{\mathbb{F}}_{p}$-points, $\pi_{2}^{-1} \circ \pi_{1}$ coincides with the map in (2) of 4.2.2 and thus factors through $\mathrm{RZ}_{L, b}$. On the completion $\left(\widehat{\mathrm{RZ}_{G, b}}\right)_{x}$ at $x \in \mathrm{RZ}_{X, G}\left(\overline{\mathbb{F}}_{p}\right)$, we get a map

$$
\operatorname{Def}_{X_{x}, G} \longrightarrow \operatorname{Def}_{X_{x_{1}}, \widetilde{L}_{1}} \times \operatorname{Def}_{X_{x_{2}}, \widetilde{L}_{2}} \simeq \operatorname{Def}_{X_{x_{1}} \times X_{x_{2}}, \widetilde{L}_{1} \times \widetilde{L}_{2}}=\operatorname{Def}_{X_{x}, \widetilde{L}}
$$

induced by the association $\mathscr{X}_{x} \mapsto\left(\mathscr{X}_{x} / \mathscr{X}_{x}^{(2)}\right) \times \mathscr{X}_{x}^{(2)}$. Note that $\left(\mathscr{X}_{x} / \mathscr{X}_{x}^{(2)}\right) \times \mathscr{X}_{x}^{(2)}$ is a deformation of $X_{x}$ via the isomorphism $\alpha_{1} \times \alpha^{(2)}$ in (5) of 4.2.2. Since this isomorphism is induced by $\alpha$, we see that $\left(\mathscr{X}_{x} / \mathscr{X}_{x}^{(2)}\right) \times \mathscr{X}_{x}^{(2)}$ lifts the tensors that define $G$-structure on $X_{x}$. Hence the image of the above map must lie in $\operatorname{Def}_{X_{x}, \widetilde{L}} \cap \operatorname{Def}_{X_{x}, G}=\operatorname{Def}_{X_{x}, L}$. 
Finally, we easily see that $\pi_{1}$ is a fibration in balls. In fact, for any point $x \in \mathrm{RZ}_{L, b}\left(\overline{\mathbb{F}}_{p}\right)$ the completion of $\mathrm{RZ}_{P, b}$ at $s(x)$ is isomorphic to a formal deformation space of $X_{x}$ with Tate tensors, which is isomorphic to a formal spectrum of a power series ring over $\breve{\mathbb{Z}}_{p}$ as proved in [Fal99], $\S 7$ (see also [Mo98], §4.).

Proposition 4.3.2. For any admissible l-adic representation $\rho$ of $J_{b}\left(\mathbb{Q}_{p}\right)$, we have

$$
H^{\bullet}\left(R Z_{L, b}^{\infty}\right)_{\rho}=H^{\bullet}\left(R Z_{P, b}^{\infty}\right)_{\rho}
$$

as virtual representations of $P\left(\mathbb{Q}_{P}\right) \times W_{E}$.

Proof. For any open compact subgroups $K_{p}{ }^{\prime} \subseteq P\left(\mathbb{Z}_{p}\right)$, we get morphisms of rigid analytic spaces

$$
s_{K_{p}{ }^{\prime}}: \mathrm{RZ}_{L, b}^{K_{p^{\prime}} \cap L\left(\mathbb{Q}_{p}\right)} \longrightarrow \mathrm{RZ}_{P, b}^{K_{p}{ }^{\prime}} \quad \text { and } \quad \pi_{1, K_{p}{ }^{\prime}}: \mathrm{RZ}_{P, b}^{K_{p}{ }^{\prime}} \longrightarrow \mathrm{RZ}_{L, b}^{K_{p}{ }^{\prime} \cap L\left(\mathbb{Q}_{p}\right)}
$$

which are $P\left(\mathbb{Q}_{p}\right) \times J_{b}\left(\mathbb{Q}_{p}\right)$-equivariant and compatible with the Weil descent datum. Moreover, $s_{K_{p}}{ }^{\prime}$ s are closed immersions and satisfy $\pi_{1, K_{p}{ }^{\prime}} \circ s_{K_{p}{ }^{\prime}}=\mathrm{id}_{\mathrm{RZ}_{L, b}^{K p^{\prime} \cap L\left(\mathbb{Q}_{p}\right)}}$.

Recall that we have a universal $p$-divisible group $\mathcal{X}_{\widetilde{P}, b}$ over $\mathrm{RZ}_{X, \widetilde{P}}$ with the associated filtration $\mathcal{X}_{\tilde{P}, b}^{\bullet}$. By [Man08, Proposition 30, we have a formal scheme $\mathrm{RZ}_{\widetilde{P}, b}^{(m)} \longrightarrow \mathrm{RZ}_{\widetilde{P}, b}$ for each integer $m>0$ with the following properties:

(i) a morphism $f: \operatorname{Spf}(R) \longrightarrow \mathrm{RZ}_{\widetilde{P}, b}$ for some $R \in \mathrm{Nilp}_{\breve{\mathbb{Z}}_{p}}$ factors through $\mathrm{RZ}_{\widetilde{P}, b}^{(m)}$ if and only if the filtration $f^{*} \mathcal{X}_{\tilde{P}, b}^{\bullet}\left[p^{m}\right]$ is split,

(ii) the formal schemes $\mathrm{RZ}_{\widetilde{P}, b}^{(m)}$ and $\mathrm{RZ}_{\widetilde{P}, b}$ become isomorphic when considered as formal schemes over $\mathrm{RZ}_{\widetilde{L}, b}$ via the map $\widetilde{\pi}_{1}: \mathrm{RZ}_{\widetilde{P}, b} \longrightarrow \mathrm{RZ}_{\widetilde{L}, b}$.

Taking the pull back of $\mathrm{RZ}_{\widetilde{P}, b}^{(m)}$ over $\mathrm{RZ}_{P, b}$, we obtain a formal scheme $\mathrm{RZ}_{P, b}^{(m)} \longrightarrow \mathrm{RZ}_{P, b}$ for each integer $m>0$ with analogous properties. We write $\mathrm{RZ}_{P, b}^{(m) \text {,rig }}$ for the rigid analytic generic fiber of $\mathrm{RZ}_{P, b}^{(m)}$.

For each integer $m>0$, we set $K_{p}{ }^{\prime(m)}:=\operatorname{ker}\left(P\left(\mathbb{Z}_{p}\right) \rightarrow P\left(\mathbb{Z}_{p} / p^{m} \mathbb{Z}_{p}\right)\right)$ and define two distinct covers $\mathcal{P}_{m} \longrightarrow \mathrm{RZ}_{P, b}^{(m)}$ and $\mathcal{P}_{m}^{\prime} \longrightarrow \mathrm{RZ}_{P, b}^{(m)}$ by the following Cartesian diagrams:

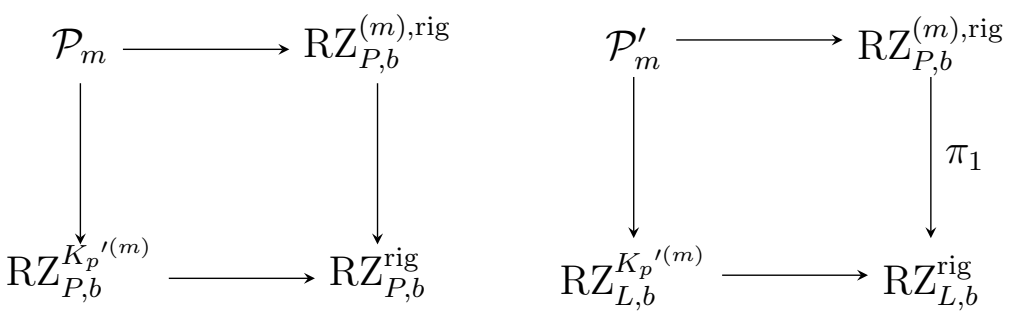

Since $\pi_{1}$ is a fibration in balls, we obtain quasi-isomorphisms

$$
R \Gamma_{c}\left(\mathcal{P}_{m}^{\prime} \otimes_{\breve{\mathbb{Q}}_{p}} \mathbb{C}_{p}, \overline{\mathbb{Q}}_{l}\right) \cong R \Gamma_{c}\left(\mathrm{RZ}_{L, b}^{K_{p}^{\prime(m)}} \otimes_{\breve{\mathbb{Q}}_{p}} \mathbb{C}_{p}, \overline{\mathbb{Q}}_{l}(-D)\right)[-2 D] \quad \text { for all } m>0
$$


where $D=\operatorname{dim} \mathrm{RZ}_{P, b}-\operatorname{dim} \mathrm{RZ}_{L, b}$. Moreover, we can argue as in [Man08, Lemma 31 and Proposition 32 to deduce quasi-isomorphisms

$$
R \Gamma_{c}\left(\mathrm{RZ}_{P, b}^{K_{p}^{\prime(m)}} \otimes_{\breve{\mathbb{Q}}_{p}} \mathbb{C}_{p}, \overline{\mathbb{Q}}_{l}\right) \cong R \Gamma_{c}\left(\mathcal{P}_{m}^{\prime} \otimes_{\breve{\mathbb{Q}}_{p}} \mathbb{C}_{p}, \overline{\mathbb{Q}}_{l}\right) \quad \text { for all } m>0
$$

Thus we have quasi-isomorphisms

$$
R \Gamma_{c}\left(\mathrm{RZ}_{P, b}^{K_{p}{ }^{\prime(m)}} \otimes_{\breve{\mathbb{Q}}_{p}} \mathbb{C}_{p}, \overline{\mathbb{Q}}_{l}\right) \cong R \Gamma_{c}\left(\mathrm{RZ}_{L, b}^{K_{p}{ }^{\prime(m)}} \otimes_{\breve{\mathbb{Q}}_{p}} \mathbb{C}_{p}, \overline{\mathbb{Q}}_{l}(-D)\right)[-2 D] \quad \text { for all } m>0
$$

which yield the desired equality.

Proposition 4.3.3. For any admissible l-adic representation $\rho$ of $J_{b}\left(\mathbb{Q}_{p}\right)$, we have

$$
H^{\bullet}\left(R Z_{G, b}^{\infty}\right)_{\rho}=\operatorname{Ind}_{P\left(\mathbb{Q}_{p}\right)}^{G\left(\mathbb{Q}_{p}\right)} H^{\bullet}\left(R Z_{P, b}^{\infty}\right)_{\rho}
$$

as virtual representations of $P\left(\mathbb{Q}_{P}\right) \times W_{E}$.

Proof. For any open compact subgroup $K_{p} \subseteq G\left(\mathbb{Z}_{p}\right)$, we have natural morphisms of rigid analytic spaces

$$
\pi_{2, K_{p}}: \mathrm{RZ}_{P, b}^{K_{p} \cap P\left(\mathbb{Q}_{p}\right)} \longrightarrow \mathrm{RZ}_{G, b}^{K_{p}}
$$

which are $P\left(\mathbb{Q}_{p}\right) \times J_{b}\left(\mathbb{Q}_{p}\right)$-equivariant and compatible with the Weil descent datum. Moreover, these maps are evidently closed immersions. Hence we have isomorphisms

$$
\mathrm{RZ}_{G, b}^{K_{p}} \cong \mathrm{RZ}_{G, b}^{K_{p}} \times_{\mathrm{RZ}}^{\text {rig }} \mathrm{RZ}_{P, b}^{\mathrm{rig}} \cong \coprod_{K_{p} \backslash G\left(\mathbb{Q}_{p}\right) / P\left(\mathbb{Q}_{p}\right)} \mathrm{RZ}_{P, b}^{K_{p} \cap P\left(\mathbb{Q}_{p}\right)} \quad \text { for all } K_{p} \subseteq G\left(\mathbb{Z}_{p}\right),
$$

thereby obtaining the desired identity.

Proposition 4.3.2 and 4.3.3 together imply Theorem 4.1.5.

\section{REFERENCES}

[Ber96] P. Berthelot, Cohomologie rigide et cohomologie rigide á support propre. premiére partie, Prépublication IRMAR 96-03(1996)

[Boy99] P. Boyer, Mauvaise réduction des variétés de Drinfeld et correspondance de Langlands locale, Invent. Math. 138(1999), 573-629.

[Fal99] G. Faltings, Integral crystalline cohomology over very ramified valuation rings, J. Amer. Math. Soc. 12 (1999), no.1, 117-144.

[Ga10] Q. Gashi, On a conjecture of Kottwitz and Rapoport, Ann. Sci. Éc. Norm. Sup. 43 (2010), 1017-1038.

[Han16] D. Hansen, Moduli of local shtuka and Harris's conjecture I, Preprint (2016)

[Har00] M. Harris, Local Langlands correspondences and vanishing cycles on Shimura varieties, European Congress of Mathematics, Vol. I(2000), 407-427.

[HT01] M. Harris, R. Taylor, On the geometry and cohomology of some simple Shimura varieties, Annals of Math. Studies, 151(2001)

[Hong16] S. Hong, On the Hodge-Newton filtration of p-divisible groups of Hodge type, Preprint, arXiv: $1606.06398(2016)$

[Ka79] N. Katz, Slope filtration of F-crystals, Astérisque 63 (1979), 113-164.

[Ki10] M. Kisin, Integral models for Shimura varieties of abelian type, J. Amer. Math. Soc. 23(4) (2010), 967-1012.

[Kim13] W. Kim, Rapoport-Zink spaces of Hodge type, Preprint, arXiv:1308.5537 (2013)

[Ko85] R. Kottwitz, Isocrystals with additional structure, Comp. Math. 56 (1985), 201-220.

[KR03] R. Kottwitz, M. Rapoport, On the existence of F-crystals, Comm. Math. Helv. 78 (2003), 153-184. 
[Lu04] C. Lucarelli, A converse to Mazurs inequality for split classical groups, J. Inst. Math. Jussieu (2004), 165-183.

[Man08] E. Mantovan, On non-basic Rapoport-Zink spaces, Ann. Sci. Éc. Norm. Sup. 41(5) (2008), 671-716.

[Mo98] B. Moonen, Models of Shimura varieties in mixed characteristics, Galois Representations in Arithmetic Algebraic Geometry, London Math. Soc., Lecture Notes Series 254, Cambridge Univ. Press (1998), 271-354

[Rap94] M. Rapoport, Non-Archimedean period domains, Proceedings of the International Congress of Mathematicians, Vol. 1, 2(1994), 423-434.

[RR96] M. Rapoport, M. Richartz, On the classification and specialization of F-isocrystals with additional structure, Comp. Math. 103(1996), 153-181.

[RV14] M. Rapoport, E. Viehmann, Towards a theory of local Shimura varieties, Münster J. Math. 7(2014), 273-326.

[RZ96] M. Rapoport, T. Zink, Period spaces for p-divisible groups, Annals of Math. Studies. 14(1996)

[Sch14] P. Scholze, p-adic geometry, Preprint (2014).

[Sh13] X. Shen, On the Hodge-Newton filtration for p-divisible groups with additional structures, Int. Math. Res. Not. no. 13, 3582-3631 (2014)

Department of Mathematics, California Institute of Technology

E-mail address: shong2@caltech.edu 